\title{
INTEGRAL IBIS, SPI, and JEM-X observations of LVT151012
}

\author{
V. Savchenko ${ }^{1,2}$, A. Bazzano ${ }^{4}$, E. Bozzo ${ }^{2}$, S. Brandt ${ }^{5}$, J. Chenevez ${ }^{5}$, T. J.-L. Courvoisier ${ }^{2}$, R. Diehl ${ }^{6}$, C. Ferrigno ${ }^{2}$, \\ L. Hanlon ${ }^{7}$, A. von Kienlin ${ }^{6}$, E. Kuulkers ${ }^{8}$, P. Laurent ${ }^{1,9}$, F. Lebrun ${ }^{9}$, A. Lutovinov ${ }^{10,11}$, A. Martin-Carrillo ${ }^{7}$, \\ S. Mereghetti ${ }^{3}$, L. Natalucci ${ }^{4}$, J. P. Roques ${ }^{12}$, T. Siegert ${ }^{6}$, R. Sunyaev ${ }^{10,13}$, and P. Ubertini ${ }^{4}$
}

1 APC, AstroParticule et Cosmologie, Université Paris Diderot, CNRS/IN2P3, CEA/Irfu, Observatoire de Paris Sorbonne Paris Cité, 10 rue Alice Domont et Léonie Duquet, 75205 Paris Cedex 13, France e-mail: Volodymyr.Savchenko@unige.ch

2 ISDC, Department of astronomy, University of Geneva, chemin d'Écogia, 16, 1290 Versoix, Switzerland

3 INAF, IASF-Milano, via E.Bassini 15, 20133 Milano, Italy

4 INAF - Institute for Space Astrophysics and Planetology, via Fosso del Cavaliere 100, 00133 Rome, Italy

5 DTU Space, National Space Institute Elektrovej, Building 327, 2800 Kongens Lyngby, Denmark

6 Max-Planck-Institut für Extraterrestrische Physik, Garching, Germany

7 Space Science Group, School of Physics, University College Dublin, Belfield, Dublin 4, Ireland

8 European Space Research and Technology Centre (ESA/ESTEC), Keplerlaan 1, 2201 AZ Noordwijk, The Netherlands

9 DSM/Irfu/Service d'Astrophysique, Bât. 709 Orme des Merisiers CEA Saclay, 91191 Gif-sur-Yvette Cedex, France

10 Space Research Institute of Russian Academy of Sciences, Profsoyuznaya 84/32, 117997 Moscow, Russia

11 Moscow Institute of Physics and Technology, Institutskiy per. 9, Dolgoprudny, Moscow Region 141700, Russia

12 Université Toulouse, UPS-OMP, CNRS, IRAP, 9 avenue Roche, BP 44346, 31028 Toulouse, France

13 Max Planck Institute for Astrophysics, Karl-Schwarzschild-Str. 1, 85741 Garching bei Munchen, Germany

14 European XFEL GmbH, Albert-Einstein-Ring 19, 22761, Hamburg, Germany

\begin{abstract}
During the first observing run of LIGO, two gravitational wave events and one lower-significance trigger (LVT151012) were reported by the LIGO/Virgo collaboration. At the time of LVT151012, the INTErnational Gamma-Ray Astrophysics Laboratory (INTEGRAL) was pointing at a region of the sky coincident with the high localization probability area of the event and thus permitted us to search for its electromagnetic counterpart (both prompt and afterglow emission). The imaging instruments on board INTEGRAL (IBIS/ISGRI, IBIS/PICsIT, SPI, and the two JEM-X modules) have been exploited to attempt the detection of any electromagnetic emission associated with LVT151012 over three decades in energy (from $3 \mathrm{keV}$ to $8 \mathrm{MeV}$ ). The omni-directional instruments on board the satellite, i.e., the SPI-ACS and the IBIS/Veto, complemented the capabilities of the IBIS/ISGRI and IBIS/PICsIT for detections outside their imaging field of view in order to provide an efficient monitoring of the entire LVT151012 localization region at energies above $75 \mathrm{keV}$. We did not find any significant transient source that was spatially and/or temporally coincident with LVT151012, obtaining tight upper limits on the associated hard X-ray and $\gamma$-ray radiation. For typical spectral models, the upper limits on the fluence of the emission from any $1 \mathrm{~s} \mathrm{counterpart} \mathrm{of} \mathrm{LVT151012} \mathrm{ranges} \mathrm{from} F_{\gamma}=3.5 \times 10^{-8} \mathrm{erg} \mathrm{cm}^{-2}(20-200 \mathrm{keV})$, within the field of view of the imaging instruments, to $F_{\gamma}=7.1 \times 10^{-7} \mathrm{erg} \mathrm{cm}^{-2}(75-2000 \mathrm{keV})$, considering the least favorable location of the counterpart for a detection by the omni-directional instruments. These results can be interpreted as a tight constraint on the ratio of the isotropic equivalent energy released in the electromagnetic emission to the total energy of the gravitational waves: $E_{75-2000 \mathrm{keV}} / E_{\mathrm{GW}}<4.4 \times 10^{-5}$. Finally, we provide an exhaustive summary of the capabilities of all instruments on board INTEGRAL to hunt for $\gamma$-ray counterparts of gravitational wave events, exploiting both serendipitous and pointed follow-up observations. This will serve as a reference for all future searches.
\end{abstract}

Key words. gravitational waves - black hole physics - X-rays: bursts - instrumentation: detectors

\section{Introduction}

Gravitational waves (GWs) were predicted as a natural consequence of general relativity (Einstein 1916), but until recently only indirect evidence of their existence had been found by measuring the time evolution of orbital parameters in binary pulsars (Hulse \& Taylor 1975; Kramer et al. 2006). The first direct detection of GWs was achieved with the discovery of GW150914 (Abbott et al. 2016d) during the first science run of the Advanced LIGO interferometer $(\mathrm{O} 1)$. This was followed by the observation of another high-significance event, GW151226 (Abbott et al. 2016b).

A third possible GW event, LVT151012, was detected during O1 with a low statistical significance of $1.7 \sigma$ or about $4.5 \%$ in the $\mathrm{O} 1$ (corresponding to a false alarm rate of once in $2.7 \mathrm{yr}$; Abbott et al. 2016a). The trigger occurred on UTC 2015-1012 09:54:43, and had a signal-to-noise ratio $(\mathrm{S} / \mathrm{N})$ of 9.7 (compared to 13 for GW151226 and 23.7 for GW150914). Considering the relatively low detection significance, this event has not been confidently classified by the LIGO/Virgo collaboration 
as a GW burst, but rather as a LIGO/Virgo Transient, possibly associated with an astrophysical event. In this case, it would correspond to a merger of two black holes of $23_{-6}^{+18} M_{\odot}$ and $13_{-5}^{+4} M_{\odot}$ at a distance of $1000_{-500}^{+500} \mathrm{Mpc}$ (Abbott et al. 2016a). LVT151012 has been localized within an area of about $1600 \mathrm{deg}^{2}$ (90\% confidence level), consisting of two elongated regions spanning over 60 degrees each. Owing to their characteristic shapes, we refer to them in the following as the southern and northern arc LIGO regions.

Electromagnetic counterparts of $\mathrm{GW}$ events are mainly expected if at least one neutron star is involved (see, e.g., Voss \& Tauris 2003; Siellez et al. 2013; Patricelli et al. 2016). However, it cannot be excluded that the merging of black holes could produce an electromagnetic signal under particular conditions (see Sect. 4). To promote the searches for possible electromagnetic counterparts of $\mathrm{GW}$ events, the LIGO/Virgo collaboration distributes near real time alerts to selected teams who have signed a memorandum of understanding. These alerts contain a localization probability map for each event and have so far led to a massive followup campaign of GW150914 and GW151226 (Abbott et al. 2016c,e; Racusin et al. 2017; Yoshida et al. 2017; Gando et al. 2016; Abe et al. 2016; Adriani et al. 2016; Cowperthwaite et al. 2016; The Pierre Auger Collaboration et al. 2016). In the case of LVT151012, the localization was distributed to the community only 6 months after the event. No extensive follow-up observations have thus been performed, and only serendipitous data sets are available from all relevant facilities.

The INTErnational Gamma-Ray Astrophysics Laboratory (INTEGRAL; Winkler et al. 2003) team participated in the search for electromagnetic counterparts of GW150914 and provided the tightest upper limits for the whole LIGO localization region above $75 \mathrm{keV}$, challenging the reported evidence for a contemporaneous $\gamma$-ray event originated from the merging of two black holes (Savchenko et al. 2016; Connaughton et al. 2016; Greiner et al. 2016). At the time of GW151226, INTEGRAL was not taking scientific data, due to the proximity of one of the perigee passages ${ }^{1}$. Slightly before and after each of these passages, all instruments on board INTEGRAL are switched off to prevent damage while crossing the Earth's radiation belts, thus limiting the observational efficiency of the observatory ("duty cycle") to $85 \%$.

We report in this paper the results of our serendipitous search for an electromagnetic counterpart of the third possible GW event, LVT151012. In Sect. 2, we first introduce the INTEGRAL general capabilities and then describe the observations performed at the time of LVT151012. Then, we report the upper limits obtained with each instrument on any possible hard X-ray $/ \gamma$-ray counterpart of LVT151012. We detail the characteristics of each instrument relevant for our purpose in Appendix A This section provides an extensive summary of the capabilities of all INTEGRAL instruments to perform similar searches, serving as a references for future studies of transient events. In Sect. 3, we combine the different results to achieve the most stringent upper limit on the electromagnetic emission from LVT151012. We discuss our results in the context of different models for the electromagnetic counterparts of GW events, illustrating the potential of our search for the detection of prompt and afterglowlike emission from similar sources (Sect. 4). We draw our conclusions in Sect. 5.

\footnotetext{
The INTEGRAL satellite orbital period was reduced from 3 to 2.6 sidereal days in January 2015.
}

\section{Observations}

INTEGRAL was designed to perform observations in the hard X-ray/soft $\gamma$-ray energy range over a $\sim 30 \times 30 \mathrm{deg}$ field of view (FoV) with two main instruments: IBIS, optimized for high angular resolution (Ubertini et al. 2003), and SPI, which provides high spectral resolution (Vedrenne et al. 2003). In addition, the JEM-X and OMC instruments provide simultaneous monitoring in the central part of the FoV in the soft X-ray and optical domains, respectively (Lund et al. 2003; Mas-Hesse et al. 2003). IBIS and SPI are equipped with active shields to reduce the cosmic-ray induced background. The event count rate provided by these shields can be used to detect transient $\gamma$-ray phenomena over the full sky, making the Anti-Coincidence Shield (ACS) of SPI and the Veto system of IBIS two $\gamma$-ray detectors with a competitive sensitivity for this purpose.

The observational mode of INTEGRAL foresees several pointings lasting roughly $1 \mathrm{~h}$ and with an aim position that is changed by a few degrees from one pointing to the other (known as the dithering strategy) in order minimize the systematics of the coded mask imaging. The imaging instruments allow us to reach deeper sensitivities (even though in a much more limited region of the sky) compared to the omni-directional instruments, which can monitor the entire sky, but are typically affected by a higher background featuring much less controllable variability. As we detail later, some of the imaging instruments are also able to provide detections for events occurring outside their FoVs at the expenses of imaging capabilities.

LVT151012 took place approximately $5 \mathrm{~min}$ before the end of the pointing 159700850010, the last scientific one in the INTEGRAL revolution 1597. This pointing started on 2015 October 12 at 2015-10-12 08:59:14 (UTC) and was concluded on the same day at 09:59:20 (UTC). At 09:58:48 (UTC), SPI was configured to enter the radiation belts and thus the SPI-ACS and SPI data acquisition was interrupted at 09:59:20 (UTC). In the current configuration, SPI enters stand-by mode slightly before the radiation belt passage. IBIS and JEM-X collected up to $230 \mathrm{~s}$ of additional science data during the following pointing 159700860010, but the observing conditions were not optimal, due to the enhanced background variability. We thus comment on these data separately. The aim point of the imaging instruments during the pointing 159700850010 was at $\mathrm{RA}=250.7$, Dec $=-45.9$, i.e., close to the most probable localization of LVT151012 within the southern arc LIGO region $(\mathrm{RA}=245.2$, $\operatorname{Dec}=-32.9$ ). At least for a fraction of this region (up to $20 \%$, depending on the instrument), we were able to exploit for the first time the high sensitivity of the observations obtained within the FoVs of the imaging instruments (JEM-X, IBIS/ISGRI, IBIS/PICsIT, and SPI) to search for an impulsive event and an afterglow-like emission. The FoV of JEM-X, IBIS/ISGRI, IBIS/PICsIT, and SPI cover an area of $130 \mathrm{deg}^{2}, 900 \mathrm{deg}^{2}$, $900 \mathrm{deg}^{2}$, and $1225 \mathrm{deg}^{2}$, respectively ${ }^{2}$. The remaining part of the southern arc and the northern arc of the LIGO localization region were only covered with lower sensitivity by the omnidirectional instruments and through the out-of-FoV observations with the IBIS/ISGRI and IBIS/PICsIT. We verified that at the time of the $\mathrm{GW}$ trigger the region of the sky occulted by the Earth was nearly $5 \mathrm{deg}$ in radius for INTEGRAL, but it did not overlap with any portion of the LIGO localization region of LVT151012 where the detection probability of the GW event was significantly higher than zero.

Given the different availabilities of the INTEGRAL instruments specified above, we performed two independent types

\footnotetext{
2 These FoV coverages are provided at $0 \%$ coding efficiency.
} 


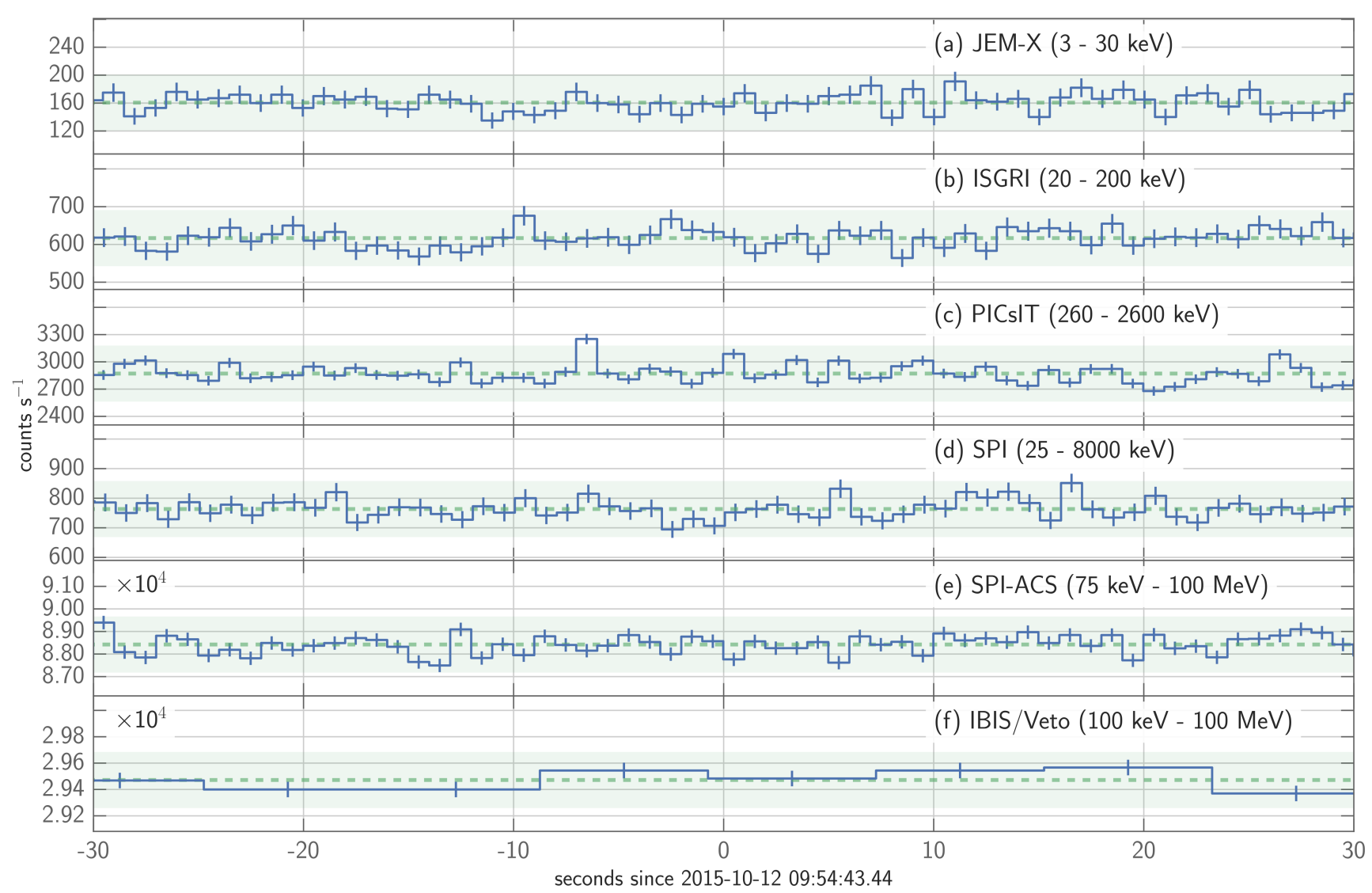

Fig. 1. Light curves obtained from the total detector count rates of different instruments on board INTEGRAL and zoomed around the time of the LVT151012 trigger provided by the LIGO/Virgo collaboration (see Sect. 1). All light curves are binned with a time resolution of $1 \mathrm{~s}$, the only exception being that obtained from the IBIS/Veto, which has an intrinsic time resolution of $8 \mathrm{~s}$ (see Sect. A.4). Also indicated are the count rates of the different instrument backgrounds (dashed lines) and the expected level of random fluctuations at $3 \sigma$ confidence level (shaded regions).

of searches in order to identify a possible $\gamma$-ray counterpart to LVT151012:

1. We searched for the prompt burst emission of LVT151012 ("impulsive event"). In this case, we exploited both the data collected by the imaging (within and outside their FoV) and omni-directional instruments in a time interval spanning from $30 \mathrm{~s}$ before to $30 \mathrm{~s}$ after the time of the LIGO trigger. Two different possibilities were considered for the spectral energy distribution of the event: (i) the hard Comptonized model that describes the typical short GRB (Gamma-Ray Burst) spectrum, parametrized by a cutoff power law with $E_{\text {peak }}=600 \mathrm{keV}$ and $\alpha=-0.5$ (these values are close to the average of the measurements obtained from all GBM short GRBs; Gruber et al. 2014); (ii) the Band model usually adopted to describe long-soft GRBs with representative parameters $E_{\text {peak }}=300 \mathrm{keV}, \alpha=-1$, and $\beta=-2.5$. We assumed the durations of $1 \mathrm{~s}$ and $8 \mathrm{~s}$ in order to be representative of the cases of short and long GRBs, respectively. The specific choice of $8 \mathrm{~s}$ binning is driven by the readout time of the IBIS/Veto light curve (see Sect. A.4).

2. We searched for the long-lasting afterglow of LVT151012. In this case, we concentrated on the detection of a hard $\mathrm{X}$-ray $/ \gamma$-ray emission in the FoV of imaging instruments that could resemble a GRB afterglow. We assumed a power-law decay of the emission intensity over time and a relatively soft spectral energy distribution $\left(F_{E} \sim E^{-2}\right)$.

In the following subsections, we describe in detail the performance of each INTEGRAL instrument for the above searches and derive separate upper limits on the flux of any possible electromagnetic counterpart of LVT151012.

\subsection{JEM-X results}

Both JEM-X modules (see also Appendix A.1) were nominally operated during the entire INTEGRAL pointing 159700850010, securing a reliable gain determination. We also verified that no particularly large background variations connected to the solar activity affected the JEM-X data collected in the time interval spanning from $300 \mathrm{~s}$ before the LVT151012 detection by LIGO to the end of the INTEGRAL pointing.

The inspection of the count rate collected by JEM-X during the $30 \mathrm{~s}$ preceding and following the onset of LVT151012 did not show any convincing presence of impulsive events (see Fig. 1a). To derive an accurate upper limit on the X-ray flux of the non-detected counterpart to the GW source, we evaluated the instrument sensitivity by using an observation of the Crab pulsar and nebula carried out earlier in the same revolution (1597). Combining the detector count rates from both JEM-X modules, we found that the limiting detectable 3-35 keV fluence of a hard $1 \mathrm{~s}$ (soft $8 \mathrm{~s}$ ) event in a location compatible with that of LVT151012 and within the instrument FoV is $4.5 \times 10^{-8} \mathrm{erg} \mathrm{cm}^{-2} \mathrm{~s}^{-1}\left(1.6 \times 10^{-7} \mathrm{erg} \mathrm{cm}^{-2} \mathrm{~s}^{-1}\right)$ at $3 \sigma$ confidence level (see also Fig. 2 for the energy dependency of the instrument sensitivity).

To search for a possible GRB afterglow-like event, we built the two JEM-X images in the time interval spanning from $30 \mathrm{~s}$ before the LIGO detection up to $287 \mathrm{~s}$ after. We made 


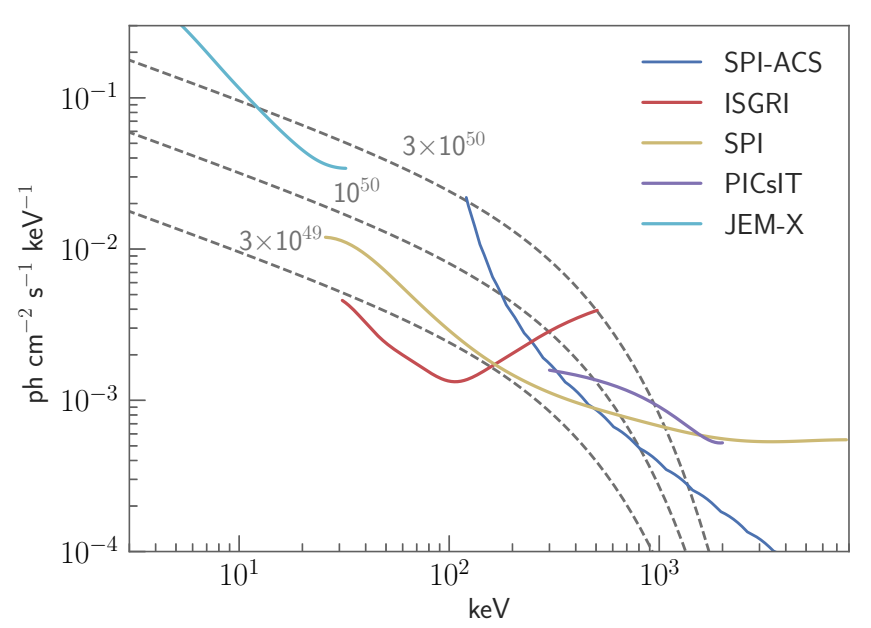

Fig. 2. Broadband continuum sensitivity $(\Delta E=E / 2)$ of the different instruments on board INTEGRAL as a function of energy for an on-axis detection of a $1 \mathrm{~s}$ impulsive event at $3 \sigma$ confidence level during the time interval spanning from $T_{0}-30 \mathrm{~s}$ to $T_{0}+30 \mathrm{~s}$. Dashed curves correspond to the case of an event at $1 \mathrm{Gpc}$ featuring a spectral energy distribution typical of a hard GRB (Comptonized model with parameters $\alpha=-0.5$, $E_{\text {peak }}=600 \mathrm{keV}$ ) and releasing a total of $3 \times 10^{49} \mathrm{erg}, 10^{50} \mathrm{erg}$, and $3 \times 10^{50}$ erg in the $75-2000 \mathrm{keV}$ energy band.

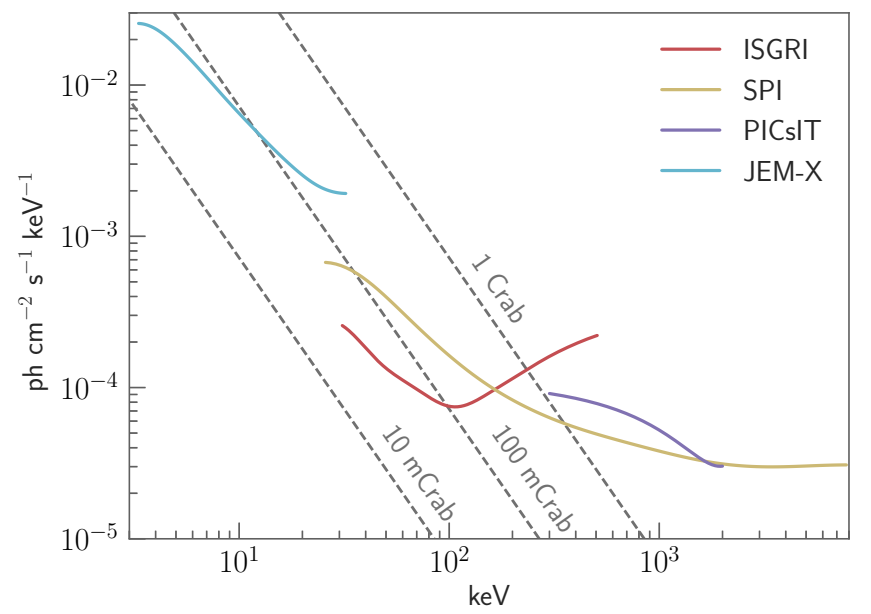

Fig. 3. Broadband continuum sensitivity $(\Delta E=E / 2)$ of the different instruments on board INTEGRAL as a function of energy for a detection of an on-axis source at $3 \sigma$ confidence level during the time interval spanning from a minimum of $T_{0}-30 \mathrm{~s}$ to a maximum of $T_{0}+287 \mathrm{~s}$ (depending on instrument). The dashed lines correspond to the case in which the source to be detected is characterized by a power-law shaped spectrum with a photon index of -2 and an X-ray flux below $100 \mathrm{keV}$ equal to $1 \mathrm{Crab}, 100 \mathrm{mCrab}$, and $10 \mathrm{mCrab}$.

use of the standard OSA software (v10.2) distributed by the ISDC (Courvoisier et al. 2003) and combined the two images to achieve the highest possible sensitivity. We did not find any convincing source in the summed image and, assuming a power-law shaped energy spectrum with $\alpha=-2$, we estimated the most stringent $3 \sigma$ upper limit on the $3-35 \mathrm{keV}$ X-ray flux of a source observed on-axis at $1.4 \times 10^{-9} \mathrm{erg} \mathrm{cm}^{-2} \mathrm{~s}^{-1}$ (see also Figs. 3 and 4 for the upper limit dependency on source position within the JEM-X FoV).

As anticipated in Sect. 2, the two JEM-X modules collected about $230 \mathrm{~s}$ of additional data in the pointing 159700860010 . An inspection of these data revealed that they were strongly affected by variable background. Thus, we do not discuss these data any further.

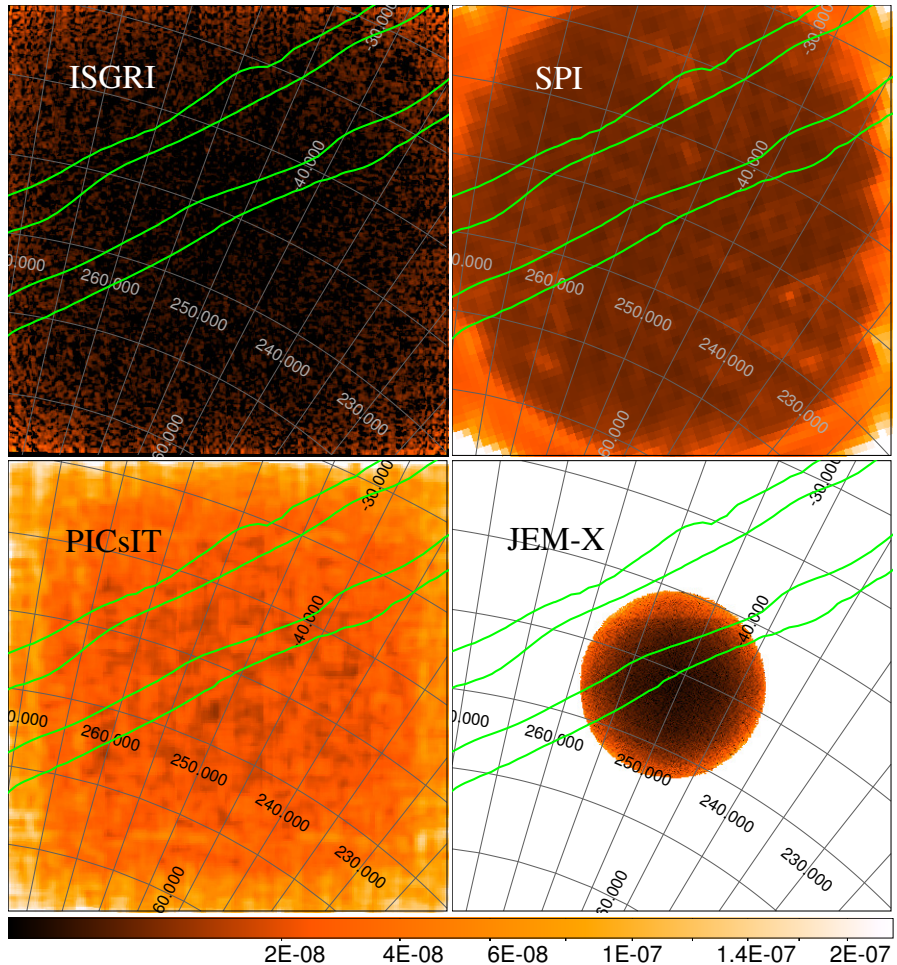

Fig. 4. Sensitivity maps within the INTEGRAL IBIS/ISGRI (20$200 \mathrm{keV}$ ), IBIS/PICsIT (260-2000 keV), SPI (25-8000 keV), and the combined JEM-X1 and JEM-X2 modules (3.-35. keV) FoVs during the time interval spanning from a minimum of $T_{0}-30 \mathrm{~s}$ to a maximum of $T_{0}+287 \mathrm{~s}$ (depending on instrument). The units reported below the color bar are $\mathrm{erg} \mathrm{cm}^{-2} \mathrm{~s}^{-1}$. We also show in green the $90 \%$ and $50 \%$ confidence level contours of the LIGO LVT151012 localization region.

\subsection{IBIS/ISGRI results}

We searched for impulsive events in the full detector light curves (neglecting the imaging capabilities, see Appendix A.2.1) covering the 20-200 keV energy range and the time interval spanning from $T_{0}-30 \mathrm{~s}$ to $T_{0}+30 \mathrm{~s}$ (where $T_{0}$ is the time of the LIGO trigger; see Fig. 1b). No significant detection was found and we estimated the most stringent $3 \sigma$ upper limit on the 20 $200 \mathrm{keV}$ flux of a $1 \mathrm{~s}(8 \mathrm{~s})$ event of $3.5 \times 10^{-8} \mathrm{erg} \mathrm{cm}^{-2} \mathrm{~s}^{-1}$ $\left(7.5 \times 10^{-8} \mathrm{erg} \mathrm{cm}^{-2} \mathrm{~s}^{-1}\right)$, assuming the localization of the event in the center of the instrument FoV and a typical hard (soft) GRB-like spectrum (see Table 1). The energy dependence of the upper limit for the $1 \mathrm{~s}$ hard event is shown in Fig. 2. ISGRI response and sensitivity coverage for events occurring outside its FoV are illustrated in Sect. 3.1 and Fig. 5 (see also Fig. A.1).

We searched for GRB afterglow-like events in the ISGRI image extracted with the OSA 10.2 software in the time interval ranging from $T_{0}-30 \mathrm{~s}$ to $T_{0}+287 \mathrm{~s}$. We did not find convincing evidence of any new source that could be associated with the GW event within the ISGRI FoV. The most stringent upper limit on the corresponding flux is derived within the instrument fully coded FoV and corresponds to $7.4 \times 10^{-10} \mathrm{erg} \mathrm{cm}^{-2} \mathrm{~s}^{-1}$ (assuming a power-law spectrum with a slope of -2 ). The dependence of ISGRI sensitivity on energy during the time interval $T_{0}-30 \mathrm{~s}-$ $T_{0}+287 \mathrm{~s}$ is shown in Fig. 3 .

About $230 \mathrm{~s}$ of additional ISGRI data were accumulated starting on 2015 October 12 during pointing 159700860010. During this time interval, the instrument was affected by larger background and we could not find any evidence of a convincing new source detection in the corresponding image. We also 
Table 1. Summary of sensitivities for the different instruments on board INTEGRAL, related to the detection of an impulsive event as the counterpart of LVT151012.

\begin{tabular}{|c|c|c|c|c|c|c|}
\hline \multirow[t]{2}{*}{ Instrument } & \multirow[t]{2}{*}{ Energy range } & \multicolumn{4}{|c|}{$3 \sigma$ sensitivity } & \multirow[b]{2}{*}{$\begin{array}{c}\text { On-axis } \\
\left(\mathrm{erg} \mathrm{cm}^{-2}\right)\end{array}$} \\
\hline & & $\begin{array}{c}\theta>120 \text { deg } \\
\left(\operatorname{erg~cm}{ }^{-2}\right)\end{array}$ & $\begin{array}{c}120 \mathrm{deg}>\theta>80 \mathrm{deg} \\
\left(\mathrm{erg} \mathrm{cm}^{-2}\right)\end{array}$ & $\begin{array}{c}80 \text { deg }>\theta>\text { FoV } \\
\left(\operatorname{erg~cm}^{-2}\right)\end{array}$ & $\begin{array}{l}\text { Field of view } \\
\left(\mathrm{erg} \mathrm{cm}^{-2}\right)\end{array}$ & \\
\hline JEM-X & $3-30 \mathrm{keV}$ & N/A & $\mathrm{N} / \mathrm{A}$ & N/A & $2.6 \times 10^{-7}, 9.2 \times 10^{-7}$ & $4.5 \times 10^{-8}, 1.6 \times 10^{-7}$ \\
\hline ISGRI & $20-200 \mathrm{keV}$ & $\mathrm{N} / \mathrm{A}$ & $\mathrm{N} / \mathrm{A}$ & $2.3 \times 10^{-7}, 1.1 \times 10^{-6}$ & $8.3 \times 10^{-8}, 1.8 \times 10^{-7}$ & $3.5 \times 10^{-8}, 7.5 \times 10^{-8}$ \\
\hline PICsIT & $260-2600 \mathrm{keV}$ & N/A & $\mathrm{N} / \mathrm{A}$ & $8.6 \times 10^{-7}, 2.4 \times 10^{-6}$ & $9.7 \times 10^{-7}, 1.9 \times 10^{-6}$ & $4.4 \times 10^{-7}, 8.7 \times 10^{-7}$ \\
\hline SPI & $25-8000 \mathrm{keV}$ & N/A & $\mathrm{N} / \mathrm{A}$ & $\mathrm{N} / \mathrm{A}$ & $1.6 \times 10^{-6}, 2.3 \times 10^{-6}$ & $3.9 \times 10^{-7}, 5.7 \times 10^{-7}$ \\
\hline SPI-ACS & $75-2000 \mathrm{keV}$ & $3.6 \times 10^{-7}, 10^{-6}$ & $2.2 \times 10^{-7}, 6 \times 10^{-7}$ & $2 \times 10^{-7}, 5.3 \times 10^{-7}$ & $3.5 \times 10^{-7}, 9.5 \times 10^{-7}$ & $4.2 \times 10^{-7}, 1.4 \times 10^{-6}$ \\
\hline IBIS/Veto & $100-2000 \mathrm{keV}$ & $8.7 \times 10^{-7}, 7.6 \times 10^{-7}$ & $2.1 \times 10^{-6}, 1.9 \times 10^{-6}$ & $1.7 \times 10^{-6}$ & $2.7 \times 10^{-6}, 2.8 \times 10^{-6}$ & $\mathrm{~N} / \mathrm{A}$ \\
\hline \multicolumn{2}{|c|}{$\begin{array}{c}\text { Fraction of the } \\
\text { LVT151012 localization }\end{array}$} & $48 \%$ & $12 \%$ & $19-36 \%$ & $4-19 \%$ & \\
\hline
\end{tabular}

Notes. Average $3 \sigma$ sensitivity for prompt events in the different regions of the sky, assuming two types of the transients: short-hard and long-soft; respectively left and right numbers. A short-hard burst is assumed to be $1 \mathrm{~s}$ long and characterized by a typical hard GRB spectrum, characterized by a Comptonized model (a parametrization of cutoff power-law power, see Gruber et al. 2014) with parameters $\alpha=-0.5, E_{\text {peak }}=600 \mathrm{keV}$. The long burst has a duration of $8 \mathrm{~s}$ and a typical soft GRB spectrum, i.e., a smoothly broken power law (Band model, Band et al. 1993) with parameters $\alpha=-1, \beta=-2.5, E_{\text {peak }}=300 \mathrm{keV}$.

checked that combining these observations with the previously used exposure resulted only in a marginally improved sensitivity. Thus, we neglected ISGRI data in pointing 159700860010 while combining the results from all INTEGRAL instruments in Sect. 3.

\subsection{IBIS/PICsIT results}

We first built the PICsIT full detector light curves in the energy range 260-2600 keV with a time resolution of $1 \mathrm{~s}$ (see Fig. 1c) and estimated the instrument background (together with its variance) to search for possible impulsive events within the time interval spanning from $T_{0}-30 \mathrm{~s}$ to $T_{0}+30 \mathrm{~s}$. A marginally significant excess ( $\mathrm{S} / \mathrm{N}$ of 3.9$)$ was found at $T_{0}-6.5 \mathrm{~s}$. The corresponding post-trial significance of this excess is 2.0 , too low to be considered a realistic detection. We thus set the $3 \sigma$ upper limit on the fluence of any $1 \mathrm{~s}(8 \mathrm{~s})$ impulsive event not detected by PICsIT within the fully coded instrument FoV at $9.7 \times 10^{-7} \mathrm{erg} \mathrm{cm}^{-2}\left(1.9 \times 10^{-6} \mathrm{erg} \mathrm{cm}^{-2}\right)$. The obtained PICsIT sensitivity as a function of energy is shown in Fig. 2. The PICsIT sensitivity to events occurring outside its FoV is illustrated in Sect. 3.1 (see also Fig. A.1).

We searched for the presence of possible long-lasting GRB afterglow-like emissions in the PICsIT images (260-2600 keV) that could be associated with LVT151012. No convincing detection was found. The corresponding results are presented in Figs. 3 and 4.

\subsection{IBIS Compton mode results}

From the measurements of the IBIS Compton-mode background in the vicinity of LVT151012, we verified that the Compton kinematics could be used to reduce by $50 \%$ the background of an on-axis source and about $75 \%$ that of a source located $40 \mathrm{deg}$ off-axis observed in this mode. A limited number of detected photons have a localization accuracy as good as $10 \%$ of the sky. However, the use of only these photons to search for possible counterparts to LVT151012 would dramatically reduce the statistics of any signal that can be recorded from any pointlike source. The highest sensitivity to the emission from a point source with an unknown position based on the Compton kinematics was obtained without applying any event selection, i.e., by using the total IBIS Compton-mode count rate.
As the effective area of IBIS Compton mode is smaller than that of both ISGRI and PICsIT, the lower number of recorded photons in this mode cannot provide by definition a more stringent upper limit on the non-detected counterpart to LVT151012 when compared to what was discussed before.

\subsection{IBIS/Veto results}

We show in Fig. 1f the IBIS/Veto light curve close to the time of the LVT151012 detection and with a bin time of $8 \mathrm{~s}$. No obvious impulsive events are found within a time interval of $\pm 30 \mathrm{~s}$ around the occurrence of LVT151012. Like the SPI-ACS, the IBIS/Veto is unable to localize the events and provide any energy resolution. Assuming the two representative GRB spectra mentioned in Sect. 2 and considering all accessible regions of interest for the IBIS/Veto, we determined $3 \sigma$ upper limits on the fluence of any impulsive events in an $8 \mathrm{~s}$ time bin ranging from $4.4 \times 10^{-7} \mathrm{erg} \mathrm{cm}^{-2}$ to $9.5 \times 10^{-6} \mathrm{erg} \mathrm{cm}^{-2}$ (see also Table 1 ).

\subsection{SPI results}

We first extracted the total instrument light curve in the 25$8000 \mathrm{keV}$ energy range with a time resolution of $1 \mathrm{~s}$ in the time interval spanning from $T_{0}-30 \mathrm{~s}$ to $T_{0}+30 \mathrm{~s}$. This light curve is shown in Fig. 1d. No clear signatures for the presence of significant impulsive events are observed.

In order to estimate the instrument sensitivity to the detection of transient sources through the coded mask imaging within the fully coded FoV, we first performed a maximum likelihood fit in each energy bin by assuming a combined contribution to the data from detector background and a single on-axis point source. We derived a $3 \sigma$ upper limit on the fluence of any nondetected $1 \mathrm{~s}$-long event during the investigated time interval of $5.7 \times 10^{-7} \mathrm{erg} \mathrm{cm}^{-2}$ in the $25-8000 \mathrm{keV}$ energy range. We show in Fig. 2 the sensitivity of SPI for similar events as a function of energy. The instrument sensitivity gradually decreases in the partially coded FoV (i.e., for off-axis angles larger than $8 \mathrm{deg}$ ), becoming null at $16 \mathrm{deg}$.

SPI images can also be used to search for GRB afterglowlike events. We could not detect any significant new source in the available image integrated from $T_{0}-30 \mathrm{~s}$ to the end of the SPI observation in INTEGRAL pointing 159700850010 $\left(T_{0}+245 \mathrm{~s}\right)$. We estimated a corresponding $3 \sigma$ upper limit 

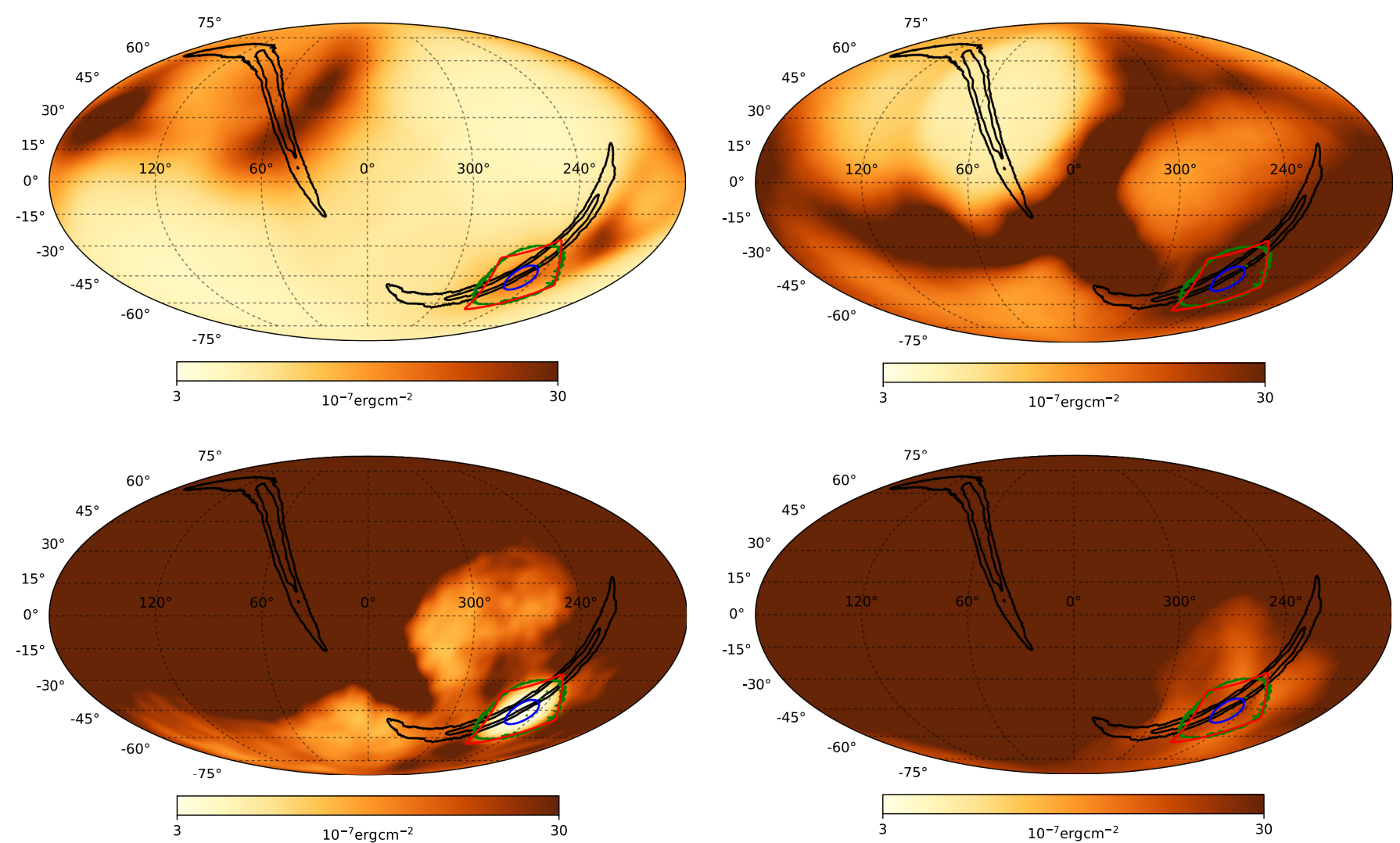

Fig. 5. Sensitivity maps of SPI-ACS, IBIS/Veto, IBIS/PICsIT, and IBIS/ISGRI for the detection of an $8 \mathrm{~s}$ soft GRB event at $3 \sigma$ confidence level whose spectral energy distribution is described by a Comptonized model with parameters $\alpha=-1$ and $\left.E_{\text {peak }}=300 \mathrm{keV}\right)$. We represent with black contours the most accurate localization of the GW event (at 50\% and 90\% confidence levels) obtained by the LALInference (Abbott et al. 2016a). Red, green, and blue contours indicate the sky regions within which the respective sensitivity of ISGRI, SPI, and JEM-X is not degrading more than a factor of 20 compared to the on-axis value.

on the 25-1000 keV X-ray flux of $2.6 \times 10^{-9} \mathrm{erg} \mathrm{cm}^{-2} \mathrm{~s}^{-1}$, assuming a power-law shaped source spectral energy distribution with a photon index of -2 and a source located within the instrument's fully coded FoV. The dependence of SPI sensitivity on energy for the detection of GRB afterglow-like events is shown in Fig. 3, while the dependence on source location within the instrument FoV is shown in Fig. 4.

\subsection{SPI-ACS results}

We extracted the full SPI-ACS detector light curve around the time of LVT151012 detection (from $T_{0}-30 \mathrm{~s}$ to $T_{0}+30 \mathrm{~s}$ ). The light curve is shown in Fig. 1e and does not display any signature of significant impulsive events above the instrumental background. For the fluence upper limit evaluation, we inspected the instrument background variability and calculated the corresponding variance in the time interval spanning from $T_{0}-300 \mathrm{~s}$ to $T_{0}+287 \mathrm{~s}$ (LVT151012 occurred close to the end of INTEGRAL orbit where the instruments are usually hit by the stream of Earth magnetospheric particles). We verified that the background variability was mainly limited to low-frequency noise $(<0.1 \mathrm{~Hz})$ in the time interval of interest. As the SPI-ACS has no energy resolution and localization capabilities, its sensitivity strongly depends on the assumptions on the source spectral energy distribution and position on the sky. Considering the two representative GRB spectra mentioned in Sect. 2 and scanning the full sky area accessible to the SPI-ACS, we determined $3 \sigma$ upper limits on the fluence of the non-detected $1 \mathrm{~s}(8 \mathrm{~s})$ event ranging from
$1.3 \times 10^{-7} \mathrm{erg} \mathrm{cm}^{-2}\left(3.3 \times 10^{-7} \mathrm{erg} \mathrm{cm}^{-2}\right)$ to $7.1 \times 10^{-7} \mathrm{erg} \mathrm{cm}^{-2}$ $\left(2.2 \times 10^{-6} \mathrm{erg} \mathrm{cm}^{-2}\right.$; see also Table 1$)$.

Evaluating SPI-ACS sensitivity as a function of the energy is particularly challenging, as it depends strongly on the spectral models considered. Assuming a number of spectra described through the GRB Band model with $\alpha$ ranging from 0 to $-2, E_{\text {peak }}$ from $50 \mathrm{keV}$ to $5 \mathrm{MeV}$, and $\beta$ from -2.5 to -5.5 , we computed the function describing the maximum photon flux at different energies that each spectrum should have in order not to be detected by the SPI-ACS in $1 \mathrm{~s}$ (at $3 \sigma$ confidence level). We use this function to plot a conservative estimate of SPI-ACS sensitivity in Fig. 2.

\section{Results}

We combine in this section all the previous results in order to derive the tightest possible upper limits on any electromagnetic emission in the entire LIGO localization region that can be associated with LVT151012, depending on the assumed spectral energy distribution and event duration.

\subsection{Impulsive events}

By exploiting the coverage of all instruments on board INTEGRAL, we were able to search for an impulsive event associated with LVT151012 in a wide energy band, covering from $3 \mathrm{keV}$ to $10 \mathrm{MeV}$. The most stringent upper limits on the detection of this event were obtained in those regions of the sky included within 


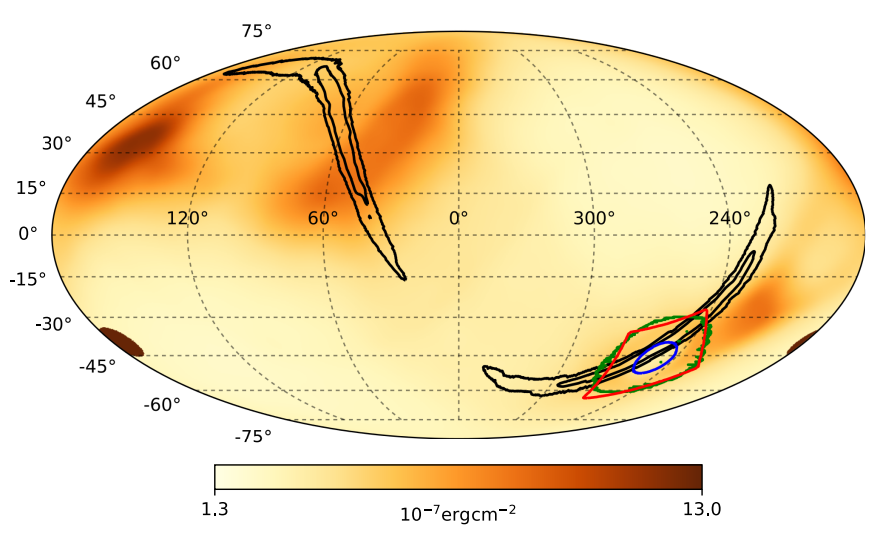

Fig. 6. Combined $3 \sigma$ upper limit on the non-detection of the electromagnetic counterpart of LVT151012 derived by using the all sky observations of SPI-ACS, IBIS/Veto, IBIS/PICsIT, and IBIS/ISGRI. The color map indicates the upper limit values in different sky regions. We assumed here the case of a $1 \mathrm{~s}$ impulsive event with a spectral energy distribution described by the Comptonized model introduced in Sect. 2. The model parameters are $\alpha=-0.5$ and $E_{\text {peak }}=600 \mathrm{keV}$. Black contours represent the most accurate localization of LVT151012 obtained from the LALInference (Abbott et al. 2016c) at both the 50\% and $90 \%$ confidence level. Red, green, and blue contours indicate the regions covered by the INTEGRAL imaging FoV observations performed with IBIS/ISGRI, SPI, and the two JEM-X modules, respectively (the larger bounds of all these FoVs has been set to the point where a worsening of the instrument sensitivity by a factor of 20 compared to the on-axis value is reached), see also Fig. 9. During this observation the Earth's shadow was near its maximum size for INTEGRAL (about 10 deg diameter) and did not occult the region of non-negligible LIGO probability.

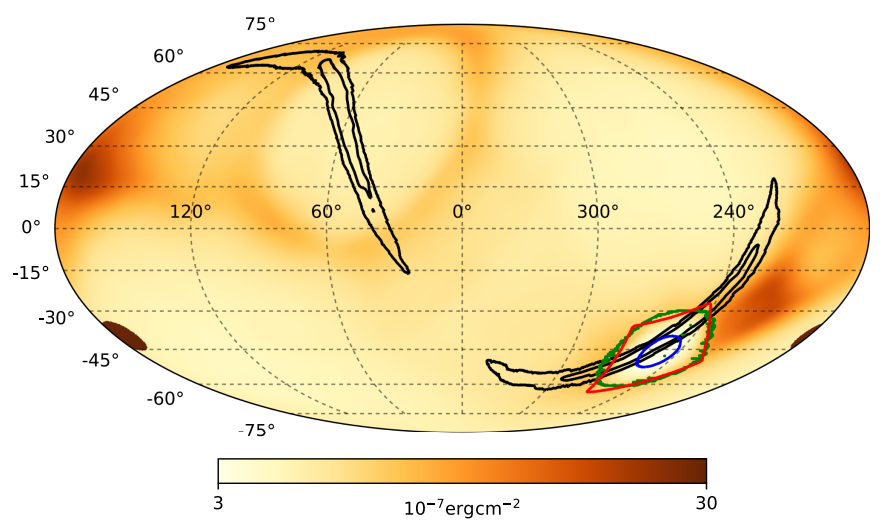

Fig. 7. Same as Fig. 6, but in the case of an $8 \mathrm{~s}$ impulsive event with a spectral energy distribution described by the Band model with parameters $\alpha=-1, \beta=-2.5$, and $E_{\text {peak }}=300 \mathrm{keV}$, see also Fig. 9 .

the imaging instruments' FoVs, containing up to about $20 \%$ of the total LIGO localization probability of LVT151012.

For the remaining regions, we could only derive upper limits on the fluence of non-detected events above $\sim 75 \mathrm{keV}$ by exploiting the INTEGRAL omni-directional instruments and the out-of-FoV coverage provided by IBIS/ISGRI and IBIS/PICsIT. An effective way of illustrating the relative capabilities of all INTEGRAL instruments for these latter observations is provided in Fig. A.1. We computed in these two panels the range of detection significances achieved by each instrument for two representative GRB-like events: a short burst ( $1 \mathrm{~s})$ characterized by a hard emission (Fig. A.1 upper panel) and a long burst (8 s) with a softer spectral energy distribution (Fig. A.1 middle panel; see also our previous definitions in Sect. 2). We assumed an arbitrary fluence for the $1 \mathrm{~s}$ or $8 \mathrm{~s}$ event and computed for each zenith angle the distribution of detection significances that can be achieved by the different instruments at all azimuthal angles. We normalized all these values to the highest detection significance obtained with the SPI-ACS and then used the derived distributions (excluding for clarity the $10 \%$ worst and $10 \%$ best values) to draw a shaded region for each instrument representing the fraction of normalized detection significance (from $0 \%$ to $100 \%$ ) as a function of the zenith angle.

We note in Fig. A.1 that the detection significance strongly depends on the zenith (i.e., off-axis) angle. Taking as a reference the connecting axis between SPI and IBIS, we can define two relevant hemispheres to explain this dependence. For the SPI-ACS, the rightmost shaded region is more extended due to the presence of IBIS, while the leftmost region is narrower as this corresponds to the unobstructed portion of the sky visible by the instrument. The regions derived in the figure for the IBIS/Veto, IBIS/ISGRI, and IBIS/PICsIT are more regular than those of the SPI-ACS as the VS: whole SPI assembly occults only a relatively small solid angle of the sky seen by IBIS. The nearly vertical red lines represent the rapid transition between high sensitivity within the IBIS/ISGRI FoV and the directions for which the passive shield of IBIS starts to be important. The different orientations of all the shaded regions highlight the complementarity of the different instruments to efficiently cover the whole sky.

Based on the above results and those obtained in Sect. 2, we conclude that in the case of short (1 s) impulsive events outside the imaging instruments FoVs, the most stringent upper limits are derived from the SPI-ACS on about $99 \%$ of the sky, ranging from $1.3 \times 10^{-7} \mathrm{erg} \mathrm{cm}^{-2}$ to $7.1 \times 10^{-7} \mathrm{erg} \mathrm{cm}^{-2}$. The IBIS/Veto is more sensitive than SPI-ACS to these events on about $1 \%$ of the sky. For impulsive events lasting more than $8 \mathrm{~s}$, IBIS/Veto is more sensitive than the SPI-ACS on about $15 \%$ of the sky (see Fig. 5). The sky fraction on which IBIS/Veto has a high detection efficiency is very reduced compared to that of SPI-ACS, but the upper limit on the non-detection of any electromagnetic emission associated with LVT151012 that can be obtained from the IBIS/Veto is a factor of 4 more stringent in those regions that are only accessible with a reduced SPI-ACS sensitivity (at $3 \sigma$ confidence level and for $8 \mathrm{~s}$ events). For events occurring inside the FoV of different instruments, ISGRI provides the most stringent upper limits for both short-hard and long-soft impulsive events, while SPI is best suited to study particularly hard events with most of the photons released at energies $\gtrsim 200 \mathrm{keV}$. JEM-X provides, in principle, coverage within the energy range $3-35 \mathrm{keV}$, but its smaller FoV compared to ISGRI makes serendipitous detections of impulsive events less likely.

The capability of INTEGRAL to constrain gamma-ray emission from the entire highly extended LIGO localization region can be efficiently illustrated by computing the LVT151012 localization probability observed with different levels of sensitivity, see Figs. 8 and 9.

Summarizing, we can identify three zones within the LIGO localization area of LVT151012 in which INTEGRAL can provide stringent upper limits on the fluence of its possible electromagnetic counterpart and which are not included within the FoVs of INTEGRAL imaging instruments:

1. off-axis angles up to $80 \mathrm{deg}$ (excluding the instruments FoVs). This region is simultaneously covered with almost the best achievable sensitivity of SPI-ACS and IBIS/ISGRI+PICsIT for out-of-FoV observations;

2. off-axis angles ranging from 80 to $120 \mathrm{deg}$. This region is covered with the best achievable sensitivity for SPI-ACS; 
Table 2. Summary of sensitivities for the different instruments on board INTEGRAL, related to the detection within their FoVs of a GRB afterglowlike event as the counterpart of LVT151012.

\begin{tabular}{cccccc}
\hline \hline Instrument & Energy range & $\begin{array}{c}\text { Field of view } \\
\mathrm{deg}^{2}\end{array}$ & $\begin{array}{c}\text { Fraction of the LVT151012 } \\
\text { localization }\end{array}$ & $\begin{array}{c}\text { Angular resolution } \\
3 \sigma \text { sensitivity } \\
\left(\mathrm{erg} \mathrm{cm}^{-2} \mathrm{~s}^{-1}\right)\end{array}$ \\
\hline JEM-X & $3-30 \mathrm{keV}$ & 110 & $4 \%$ & $3^{\prime}$ & $1.4 \times 10^{-9}$ \\
ISGRI & $20-200 \mathrm{keV}$ & 820 & $19 \%$ & $12^{\prime}$ & $7.4 \times 10^{-10}$ \\
PICsIT & $260-2600 \mathrm{keV}$ & 820 & $19 \%$ & $30^{\prime}$ & $2.4 \times 10^{-8}$ \\
SPI & $25-1000 \mathrm{keV}$ & 790 & $18 \%$ & $2.5^{\circ}$ & $4.2 \times 10^{-9}$ \\
\hline
\end{tabular}

Notes. Data were integrated within the time interval spanning from a minimum of $T_{0}-30$ to a maximum of $T_{0}+287$ (depending on the instrument) and a power-law shaped spectral energy distribution with a photon index of -2 has been assumed for the event to be detected. The limit of the FoVs has been set to the point where a worsening of the instrument sensitivity by a factor of 20 compared to the on-axis value is reached.

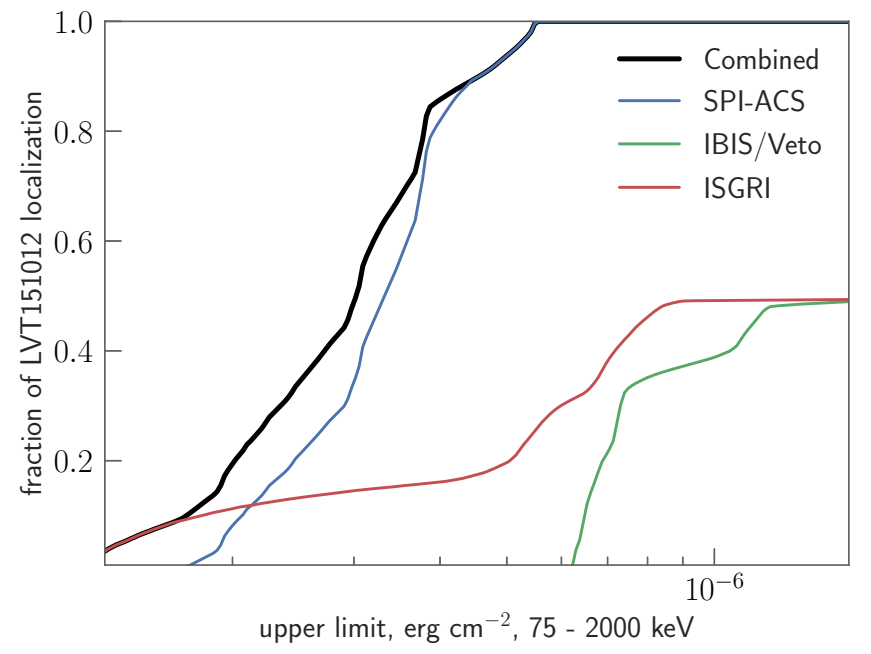

Fig. 8. Probability of LVT151012 as a function of combined and individual instrument $3 \sigma$ upper limits on the non-detection of its electromagnetic counterpart. We assumed here the case of a $1 \mathrm{~s}$ impulsive event with a spectral energy distribution described by the Comptonized model introduced in Sect. 2. The model parameters are $\alpha=-0.5$ and $E_{\text {peak }}=600 \mathrm{keV}$.

3. off-axis angles larger than $120 \mathrm{deg}$. In this case, SPI-ACS can only cover the region with limited sensitivity, but the IBIS/Veto almost achieves its best performance.

The sensitivity of different instruments for the detection of a $1 \mathrm{~s}$ or $8 \mathrm{~s}$ event in these regions is summarized in Table 1 and Fig. 2.

\subsection{GRB afterglow-like emission}

The upper limits on the non-detection of a GRB afterglow-like emission $\left(F_{E} \sim E^{-2}\right)$ from LVT151012 that we obtained inspecting the FoV of imaging instruments on board INTEGRAL (JEM-X, IBIS/ISGRI, IBIS/PICsIT, and SPI) are summarized in Table 2. ISGRI provides the highest sensitivity to search for such emissions within the IBIS FoV (see Fig. 4). Harder events with the bulk of high-energy radiation emitted above $\sim 200 \mathrm{keV}$ can be more efficiently detected by SPI. JEM-X is the only instrument providing coverage at energies $<20 \mathrm{keV}$.

These results are particularly relevant for the dedicated target of opportunity (ToO) observations that are being planned with INTEGRAL in order to follow-up future triggers provided by LIGO and Virgo. These ToO observations will image the accessible regions of the sky included in the localization of GW events within $\sim 1$ day after the trigger, thus probing mainly

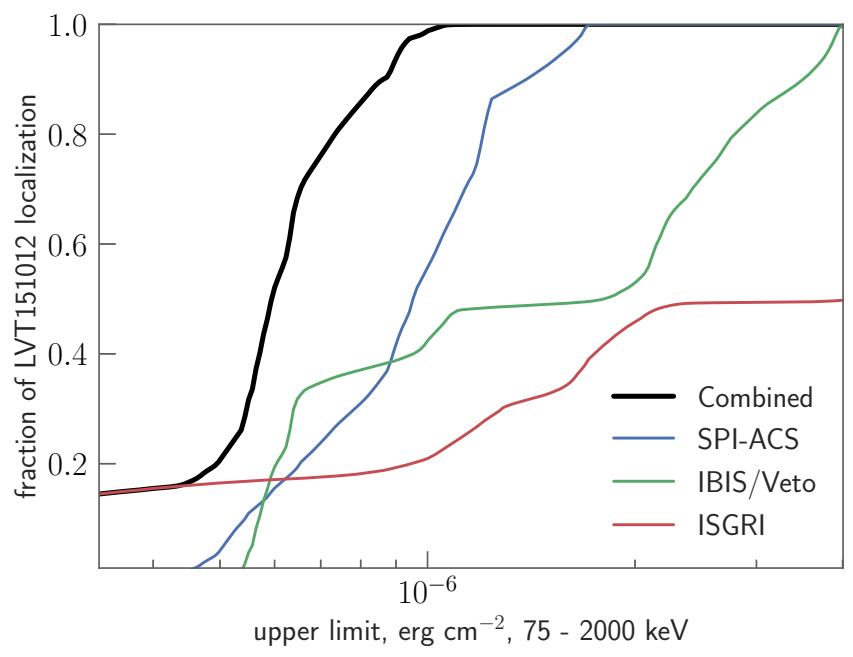

Fig. 9. Same as Fig. 8, but in the case of an $8 \mathrm{~s}$ impulsive event with a spectral energy distribution described by the Band model with parameters $\alpha=-1, \beta=-2.5$, and $E_{\text {peak }}=300 \mathrm{keV}$.

the presence of any long-lasting electromagnetic emission from the GW source. The complementary of INTEGRAL instruments provides a unique broadband coverage and is very well suited to hunting for electromagnetic counterparts of GW events.

\section{Discussion}

INTEGRAL features a duty cycle as high as $85 \%$ for the collection of scientific data, being unable to monitor the X-ray $/ \gamma$-ray sky only for a short amount of time around the perigee passage when all instruments are turned off, due to charged particles in the Earth radiation belts. Its elongated orbit strongly limits the occultation of the accessible sky by the Earth, making the suite of all available onboard instruments capable of efficiently catching the prompt emission associated with GW events and of following up any possible related afterglow in a broad energy range (3 keV-10 MeV).

SPI-ACS is the most sensitive all-sky detector of $\gamma$ rays with energies $\gtrsim 100 \mathrm{keV}$ among the instruments currently in operation or solidly planned in the foreseeable future. It can be efficiently exploited to hunt for the prompt emission associated with LIGO/Virgo triggers, and could provide in the case of LVT151012 relatively deep $3 \sigma$ upper limits on the fluence of non-detected counterparts on the whole sky, ranging from $1.3 \times 10^{-7} \mathrm{erg} \mathrm{cm}^{-2}$ to $2.2 \times 10^{-6} \mathrm{erg} \mathrm{cm}^{-2}$ (see Table 1 and Fig. 5). IBIS (IBIS/Veto, IBIS/ISGRI, and IBIS/PICsIT) 
is also able to provide monitoring of a large fraction of the sky, efficiently complementing SPI-ACS data where its sensitivity is reduced. Moreover, we showed that for the majority of transients more than one INTEGRAL all-sky instrument is able to provide significant detection, opening a possibility of constraining the signal arrival direction and its nature. Even with the current level of systematic uncertainty, in some particularly favorable cases, it is possible to narrow down the uncertainty on the event location to within $1 \%$ of the sky.

A combination of all-sky INTEGRAL detectors allows us to put stringent upper limit on the hard X-ray emission associated with LVT151012. This limit can be interpreted as a constraint on the ratio of isotropic equivalent electromagnetic energy emitted in an isolated burst during the LVT151012 to the total energy of the gravitational waves: $E_{75-2000 \mathrm{keV}} / E_{\mathrm{GW}}<$ $4.4 \times 10^{-5}\left[\frac{D}{1000 \mathrm{Mpc}}\right]^{2}\left[\frac{F_{75-2000 \mathrm{kev}}}{10^{-6} \mathrm{erg} \mathrm{cm}^{-2}}\right]\left[\frac{E_{\mathrm{GW}}}{1.5 \times M_{\odot} c^{2}}\right]^{-1}$. This constraint is less tight than the one we found in the case of GW150914 (Savchenko et al. 2016) for several reasons: the LIGO event was more distant, less luminous, localized less favorably for SPI-ACS, and took place near the end of INTEGRAL observations when the background was somewhat disturbed.

As the FoV of IBIS and SPI corresponds to only $2 \%$ of the sky, events similar to the prompt emission of GRBs are rarely observed within these limited areas (the situation is even worse for the two JEM-X modules, given the much smaller FoV than both IBIS and SPI). Although the number of correspondingly significant reported detections is relatively small (about ten detections a year for IBIS; Mereghetti 2012), the high INTEGRAL sensitivity in the hard X-ray domain efficiently probes a population of particularly faint and soft $\gamma$-ray bursts, with $20-200 \mathrm{keV}$ fluences down to a few $10^{-8} \mathrm{erg} \mathrm{cm}^{-2}$ (Bošnjak et al. 2014). If at least a fraction of the LIGO/Virgo localization area for the future GW triggers overlap with the IBIS and/or SPI FoV, INTEGRAL observations could be used to localize and characterize in fairly good detail the corresponding electromagnetic counterparts, or at least put tight constraints on their hard X-ray $/ \gamma$-ray emission (as is done in the present paper for LVT151012). The possibility of exploiting the presence of the coded masks to obtain the JEM-X, IBIS, and SPI images allows us also to perform in these cases searches for any possible GRB afterglow-like emission associated with the GW event. This will be the main goal of the deep pointed observations that INTEGRAL aims to perform within $\sim 1 \mathrm{~d}$ of the announcement of future LIGO/Virgo triggers, whenever compatible with the satellite operational constraints.

For the fraction of the LVT151012 probability region in the field of view of the INTEGRAL detectors we are able to put a tight upper limit on the long-lasting hard X-ray emission component. This implies a constraint on the ratio of isotropic equivalent electromagnetic energy emitted in the $287 \mathrm{~s}$ following the LVT151012 to total energy of the gravitational waves: $E_{75-2000 \mathrm{keV}} / E_{\mathrm{GW}}<8.9 \times 10^{-6}\left[\frac{D}{1000 \mathrm{Mpc}}\right]^{2}\left[\frac{F_{20-200 \mathrm{ke}}}{2 \times 10^{-7} \mathrm{erg} \mathrm{cm}^{-2}}\right]$ $\times\left[\frac{E_{\mathrm{GW}}}{1.5 \times M_{\odot} c^{2}}\right]^{-1}$. Here we have chosen the most suitable energy range for IBIS/ISGRI, which is the most sensitive instrument to long-lasting emission characterized by our assumed spectral energy distribution (a power law with a slope of -2 ).

The nature of the hard X-ray emission that we should expect from the likely events associated with GW remains speculative. Afterglows of long and short GRBs are among the most anticipated counterparts of the LIGO and Virgo detections, but their hard X-ray properties are not yet well understood. When a bright nearby long GRB is observed by a sensitive hard $\mathrm{X}$-ray instrument, it may remain visible for many hours. Two examples are those of GRB 990123, which was detected at energies $\gtrsim 20 \mathrm{keV}$ by BeppoSAX/PDS up to $20 \mathrm{ks}$ after the onset of the event (Corsi et al. 2005), and GRB120711A, which was observed by INTEGRAL/ISGRI (INTEGRAL/JEM-X) to become faint not earlier than $10 \mathrm{ks}$ (20 ks; Martin-Carrillo et al. 2014). The long-lasting hard X-ray emission of GRB120711A was shown to be remarkably similar to that recorded in the $\mathrm{GeV}$ band by the Fermi/LAT. As the detection of luminous GRB afterglows are not uncommon at these very high energies (Ackermann et al. 2013), it seems reasonable to expect that an associated hard X-ray emission should also be frequently observed. Convincing evidence for the presence of a slowly decaying hard X-ray and soft $\gamma$-ray afterglow emission has been reported for several GRBs, such as GRB 130427A (Maselli et al. 2014) and GRB 080319B (Savchenko 2012, Fig. 4.14). In the case of GRB 130427A, the connection between the afterglow in the hard X-ray and $\gamma$-ray bands was supported by a highly sensitive pointed NuSTAR observation (Kouveliotou et al. 2013). The paucity of previous detections at hard X-rays could be mostly ascribed to the relatively narrow FoV of the instruments available in this energy domain and the lack of performed follow-up observations of GRB afterglows.

Rescaling the hard X-ray afterglow of GRB 120711A (redshift of 1.4 or $D_{L}=10 \mathrm{Gpc}$ ) to the distance of LVT151012, which also corresponds to the typical observational horizon of the LIGO O1/O2 runs (redshift of 0.2 or $D_{L}=1 \mathrm{Gpc}$ ), would make such an event significantly detectable in ISGRI and JEM-X in the 10 days following the initial onset of the burst (we assumed here that the decay in time continued according to what was observed during the initial afterglow of GRB 120711A or, in the worst case, that it followed a power-law shaped decay in time characterized by a slope not steeper than $t^{-2}$ ). If the direction of an outflow from a GRB is not aligned with the observer's line of sight, the high-energy luminosity during an afterglow will be suppressed and will reach its peak at a later time (Granot et al. 2002). Comparing the $3 \sigma$ sensitivity of INTEGRAL/ISGRI to the luminosity of the afterglow recorded from GRB 120711A, rescaled to a distance of $1 \mathrm{Gpc}$, we found that ISGRI could have detected such event, even if the outflow was not originally pointed toward the observer, as long as the angle between the outflow direction and the line of sight was no more than 30 degrees. We note that, assuming a value of a GRB jet opening angle similar to that measured in the case of GRB 120711A, only small percent of the misaligned detections would be accompanied by a prompt emission. This suggests the presence of a considerable population of orphan hard X-ray afterglows. A similar result can be obtained for the case of GRB 130427A using the outcomes of dedicated multiwavelength observations. The hard X-ray emission associated with the afterglow of this exceptionally bright burst was observed by Swift/BAT and NuSTAR, which operate in low-Earth orbits and can point continuously to a celestial target for no longer than $\sim 3 \mathrm{ks}$. As the highly elliptical orbit of INTEGRAL enables uninterrupted observations with a duration of up to about two days, we estimated that the afterglow of GRB 130427A should have remained detectable to the INTEGRAL instruments for about 1 day following the onset of the event.

In at least a few cases, the prompt emission of the GRB was observed to last for more than 20 ks (e.g., Evans et al. 2014; Savchenko et al. 2013), and thus a very rapid hard X-ray followup could catch the burst still going off while giving rise to an associated GW emission.

Even though the above arguments show that there are promising prospects to observe the afterglows of long GRBs 
with INTEGRAL, it is presently expected that GW events should be associated with short GRBs following the merging of compact binary systems involving at least one neutron star. So far, no hard X-ray afterglows have been detected from short GRBs. Fermi/LAT observations of GRB 090510 provided some indications that a particularly bright short GRB could display a $\gamma$-ray afterglow. We conclude that the presence of a hard X-ray afterglow following at least a few short GRBs cannot be completely ruled out yet. On the other hand, it is also possible that energetic supernovae, accompanied by a bright and long GRB, produce a distinct gravitational wave signal (see Ott 2009, for a review).

Magnetars are also thought to produce GWs with sufficient intensity to be detected by Advanced LIGO and Advanced Virgo (e.g., Zink et al. 2012). Several objects in this class showed outbursts lasting for a few days during which many bright flares of hard X-rays were going off, with a clearly detectable highenergy emission extending up to $100 \mathrm{keV}$, producing remarkable signals in the INTEGRAL detectors (Mereghetti et al. 2009; Savchenko et al. 2010). In the future, GW signals possibly associated with these events could be efficiently followed up with INTEGRAL.

Future GW detections might also pave the way for still unexplored (or poorly explored) physical mechanisms to trigger substantial electromagnetic emissions during these events. This was already the case when the tentative gamma-ray counterpart of GW150914 was announced (Connaughton et al. 2016). A $\mathrm{BH}-\mathrm{BH}$ merger is expected to have little material (if any at all) in its immediate surroundings and was thus has not yet been considered a likely source of electromagnetic emissions. However, it has been speculated that even in this case a GRB-like event may be expected under some peculiar assumptions, such as the presence of a long-lived accretion disk (Perna et al. 2016), the coalescence immediately following the collapse of a star (Loeb 2016; Woosley 2016), the non-vanishing electric charge of a black hole (Fraschetti 2016), or the creation of a transient naked singularity (Malafarina \& Joshi 2016). If a binary BH merger can be associated with beamed relativistic outflows, their interaction with the close-by ambient medium might led to the production of a long-lasting electromagnetic emission observable from the radio to $\mathrm{TeV}$ energies, as happens during a GRB afterglow (Zhu \& Wang 2016).

\section{Conclusions}

At odds with the cases of GW150914 and GW151226, there was no extensive multiwavelength campaign to follow up the discovery of LVT151012 due to the late release of the corresponding announcement by the LIGO/Virgo collaboration. Luckily, INTEGRAL was pointing toward the peak of the LIGO localization probability, allowing us to exploit the full capabilities of its instruments. We reported in this paper the serendipitous search for electromagnetic counterparts to the possible GW event by exploiting all INTEGRAL data that were collected at that time. The results of our analysis provide the most sensitive constraints obtained so far on the non-detection of any electromagnetic emission associated with LVT151012 in a large fraction of the event localization, as well as very competitive limits over the whole 90\% localization region. This confirms and strengthens the potential of INTEGRAL to hunt for GW counterparts, as already demonstrated at the time of GW150914.

Using the SPI-ACS, we set a $3 \sigma$ upper limit on the whole localization region of LVT151012 ranging from $1.3 \times$ $10^{-7} \mathrm{erg} \mathrm{cm}^{-2}$ to $2.2 \times 10^{-6} \mathrm{erg} \mathrm{cm}^{-2}$ in $75-2000 \mathrm{keV}$ (depending on the source location, spectrum, and duration). This result rules out the possibility that a bright impulsive burst of $\gamma$ rays is associated with the $\mathrm{GW}$ event, constraining the ratio of the isotropic equivalent energy released in such an event to the energy of the GWs: $E_{75-2000 \mathrm{keV}} / E_{\mathrm{GW}}<4.4 \times 10^{-5}$. Additional upper limits are provided for specific limited regions in the sky, taking advantage of the complementary coverage provided by the second omni-directional instrument on board INTEGRAL, the IBIS/Veto, and the two imaging instruments capable of providing coverage also outside their FoV, i.e., the IBIS/ISGRI and IBIS/PICsIT. In particular, the data of IBIS/Veto reach a better sensitivity than those of SPI-ACS in the sky region located at off-axis angles larger than $120 \mathrm{deg}$ with respect to the satellite aim point, while ISGRI and PICsIT improve the sensitivity for locations in the sky up to $15 \mathrm{deg}$ off-axis (30 deg in case of events characterized by a particularly soft spectral energy distribution). The most stringent upper limits for impulsive events associated with LVT151012 were obtained within the FoV of the coded-mask imaging instruments IBIS, SPI, and JEM-X, which overlapped with a large portion of the higher probability localization region of the $\mathrm{GW}$ event. We set a $3 \sigma$ upper limit of $3.5 \times 10^{-8} \mathrm{erg} \mathrm{cm}^{-2}\left(7.5 \times 10^{-8} \mathrm{erg} \mathrm{cm}^{-2}\right)$ in the 20 $200 \mathrm{keV}$ energy range, $3.9 \times 10^{-7} \mathrm{erg} \mathrm{cm}^{-2}\left(5.7 \times 10^{-7} \mathrm{erg} \mathrm{cm}^{-2}\right)$ in the $25-8000 \mathrm{keV}$ energy range, and $4.5 \times 10^{-8} \mathrm{erg} \mathrm{cm}^{-2}$ $\left(1.6 \times 10^{-7} \mathrm{erg} \mathrm{cm}^{-2}\right)$ in the $3-30 \mathrm{keV}$ energy range, in the case of a short-hard (long-soft) GRBs-like impulsive event observed close to the spacecraft aim point. These results are summarized in Table 1.

The availability of images from IBIS, SPI, and JEM-X also allowed us to search in the corresponding covered fraction of the sky for GRB afterglow-like events possibly associated with LVT151012. Also in this case no significant detection was found, limiting the ratio of the isotropically equivalent energy in slowly decaying hard X-ray afterglow to total GW energy to $E_{20-200 \mathrm{keV}} / E_{\mathrm{GW}}<8.9 \times 10^{-6}$. We summarized all the results in Table 2 . For future triggers, that will be promptly announced by the LIGO/Virgo collaboration, INTEGRAL plans dedicated pointed observations that will cover as much of the event localization regions as possible with the imaging instruments to search and characterize long-lasting high-energy emission associated with GW events. The joint effort of Virgo and LIGO might also lead in the future to an enhanced localization capability for GW events that could allow INTEGRAL to cover the entire region with a single pointing.

Our non-detections of any electromagnetic counterpart to LVT151012 (to within the limiting observational capabilities of the instruments on board INTEGRAL) remains compatible so far with the commonly accepted scenario that no hard X-ray/ $\gamma$-ray emission is expected from the coalescence of a binary black hole.

Acknowledgements. This paper is based on data from observations with INTEGRAL, an ESA project with instruments and science data center funded by ESA member states (especially the PI countries: Denmark, France, Germany, Italy, Spain, and Switzerland), Czech Republic and Poland, and with the participation of Russia and the USA. The SPI-ACS detector system was provided by MPE Garching/Germany. We acknowledge the German INTEGRAL support through DLR grant 50 OG 1101. The Italian INTEGRAL/IBIS team acknowledges the support of ASI/INAF agreement No. 2016-025-R.0. A.L. and R.S. acknowledge the support from the Russian Science Foundation (grant 14-22-00271). Some of the results in this paper have been derived using the HEALPix (Górski et al. 2005) package. We are grateful the François Arago Centre at APC for providing computing resources, and VirtualData from LABEX P2IO for enabling access to the StratusLab academic cloud. Finally we thank the referee for careful reading of the manuscript and for the insightful comments. 


\section{References}

Abbott, B. P., Abbott, R., Abbott, T. D., et al. 2016a, Phys. Rev. X, 6, 041015 Abbott, B. P., Abbott, R., Abbott, T. D., et al. 2016b, Phys. Rev. Lett., 116 241103

Abbott, B. P., Abbott, R., Abbott, T. D., et al. 2016c, ApJ, 826, L13

Abbott, B. P., Abbott, R., Abbott, T. D., et al. 2016d, Phys. Rev. Lett., 116, 061102

Abbott, B. P., Abbott, R., Abbott, T. D., et al. 2016e, ApJS, 225, 8

Abe, K., Haga, K., Hayato, Y., et al. 2016, ApJ, 830, L11

Ackermann, M., Ajello, M., Asano, K., et al. 2013, ApJS, 209, 11

Adriani, O., Akaike, Y., Asano, K., et al. 2016, ApJ, 829, L20

Band, D., Matteson, J., Ford, L., et al. 1993, ApJ, 413, 281

Bošnjak, Ž., Götz, D., Bouchet, L., Schanne, S., \& Cordier, B. 2014, A\&A, 561, A25

Caballero, I., Zurita Heras, J. A., Mattana, F., et al. 2013, ArXiv e-prints [arXiv: 1304.1349]

Connaughton, V., Burns, E., Goldstein, A., et al. 2016, ApJ, 826, L6

Corsi, A., Piro, L., Kuulkers, E., et al. 2005, A\&A, 438, 829

Courvoisier, T. J.-L., Walter, R., Beckmann, V., et al. 2003, A\&A, 411, L53

Cowperthwaite, P. S., Berger, E., Soares-Santos, M., et al. 2016, ApJ, 826, L29

Einstein, A. 1916, in Sitzungsberichte der Königlich Preußischen Akad. der Wissenschaften (Berlin), 688

Evans, P. A., Willingale, R., Osborne, J. P., et al. 2014, MNRAS, 444, 250

Ferguson, C., Barlow, E. J., Bird, A. J., et al. 2003, A\&A, 411, L19

Forot, M., Laurent, P., Lebrun, F., \& Limousin, O. 2007, ApJ, 668, 1259

Fraschetti, F. 2016, ArXiv e-prints [arXiv: 1603 . 01950]

Gando, A., Gando, Y., Hachiya, T., et al. 2016, ApJ, 829, L34

Górski, K., Hivon, E., Banday, A., et al. 2005, ApJ, 622, 759

Granot, J., Panaitescu, A., Kumar, P., \& Woosley, S. E. 2002, ApJ, 570, L61

Greiner, J., Burgess, J. M., Savchenko, V., \& Yu, H.-F. 2016, ApJ, 827, L38

Gruber, D., Goldstein, A., Weller von Ahlefeld, V., et al. 2014, ApJS, 211, 12

Hulse, R., \& Taylor, J. 1975, ApJ, 195, L51

Kouveliotou, C., Granot, J., Racusin, J. L., et al. 2013, ApJ, 779, L1

Kramer, M., Stairs, I. H., Manchester, R. N., et al. 2006, Science, 314, 97

Labanti, C., Di Cocco, G., Ferro, G., et al. 2003, A\&A, 411, L149

Laurent, P., Limousin, O., Cadolle-Bel, M., et al. 2003, A\&A, 411, L179

Lebrun, F., Leray, J. P., Lavocat, P., et al. 2003, A\&A, 411, L141
Loeb, A. 2016, ApJ, 819, L21

Lund, N., Budtz-Jørgensen, C., Westergaard, N. J., et al. 2003, A\&A, 411, L231

Malafarina, D., \& Joshi, P. S. 2016, ArXiv e-prints [arXiv: 1603. 02848]

Marcinkowski, R., Denis, M., Bulik, T., et al. 2006, A\&A, 452, 113

Marcinkowski, R., Denis, M., Bulik, T., et al. 2007, in ESA Spec. Publ., 622, 609

Martin-Carrillo, A., Hanlon, L., Topinka, M., et al. 2014, A\&A, 567, A84

Maselli, A., Melandri, A., Nava, L., et al. 2014, Science, 343, 48

Mas-Hesse, J. M., Giménez, A., Culhane, J. L., et al. 2003, A\&A, 411, L261

Mereghetti, S. 2012, in Pos (INTEGRAL 2012)007

Mereghetti, S., Götz, D., Weidenspointner, G., et al. 2009, ApJ, 696, L74

Ott, C. D. 2009, Class. Quant. Grav., 26, 063001

Patricelli, B., Razzano, M., Cella, G., et al. 2016, J. Cosmol. Astropart. Phys., 11,056

Perna, R., Lazzati, D., \& Giacomazzo, B. 2016, ApJ, 821, L18

Quadrini, E. M., Bazzano, A., Bird, A. J., et al. 2003, A\&A, 411, L153

Racusin, J. L., Burns, E., Goldstein, A., et al. 2017, ApJ, 835, 82

Savchenko, V. 2012, Gamma-ray bursts, Ph.D. Thesis, Université de Genève, Switzerland

Savchenko, V., Neronov, A., Beckmann, V., Produit, N., \& Walter, R. 2010, A\&A, 510, A77

Savchenko, V., Beckmann, V., Ferrigno, C., et al. 2013, GRB Coordinates Network, 15259

Savchenko, V., Ferrigno, C., Mereghetti, S., et al. 2016, ApJ, 820, L36

Siellez, K., Boer, M., \& Gendre, B. 2013, MNRAS, 437, 649

Sturner, S., Shrader, C., Weidenspointner, G., et al. 2003, A\&A, 411, L81

The Pierre Auger Collaboration, Aab, A., Abreu, P., et al. 2016, Phys. Rev. D 94, 122007

Ubertini, P., Lebrun, F., Di Cocco, G., et al. 2003, A\&A, 411, L131

Vedrenne, G., Roques, J.-P., Schönfelder, V., et al. 2003, A\&A, 411, L63

von Kienlin, A., Beckmann, V., Rau, A., et al. 2003, A\&A, 411, L299

Voss, R., \& Tauris, T. M. 2003, MNRAS, 342, 1169

Winkler, C., Courvoisier, T. J.-L., di Cocco, G., et al. 2003, A\&A, 411, L1

Woosley, S. E. 2016, ApJ, 824, L10

Yoshida, M., Utsumi, Y., Tominaga, N., et al. 2017, PASJ, 69, 9

Zhu, Q.-Y., \& Wang, X.-Y. 2016, ApJ, 828, L4

Zink, B., Lasky, P. D., \& Kokkotas, K. D. 2012, Phys. Rev. D, 85, 024030 


\section{Appendix A: Instrument description}

\section{A.1. JEM-X}

JEM-X is a coded-mask X-ray monitor consisting of two identical Microstrip Gas Chamber modules which have been simultaneously operational since 2010 (Lund et al. 2003). The two modules are surrounded by a collimator tube that is nearly opaque to $\mathrm{X}$-rays between 3 and $35 \mathrm{keV}$, where JEM-X is collecting science data. For this reason, the instrument is not useful to search for impulsive events occurring outside its FoV. As JEM-X is endowed with a relatively large FoV, featuring a diameter of $13.2 \mathrm{deg}$ at zero response, and provides coverage in an energy range that is not accessible by any other INTEGRAL instrument, it is particularly well suited and best exploited to search for long-lasting soft X-ray transients. The extraction of reliable measurements from the JEM-X science data is not always straightforward as the response of the detector is occasionally unstable during the observations, owing to temperature changes, irregularities introduced by detector cold start, or autonomous switchoffs and reactivations.

\section{A.2. IBIS}

IBIS is a hard X-ray telescope characterized by a relatively good angular resolution of about 12 arcmin and a point source location accuracy (PSLA) of 1-3 arcmin (for sources with typical fluxes of a few mCrab in the $20-40 \mathrm{keV}$ energy range). The reconstruction of sky images is performed by exploiting the availability of a coded mask located $3.2 \mathrm{~m}$ above the detection plane at the extreme end of the telescope. This results in a $9 \times 9$ degree fully coded FoV, extending to about $30 \times 30$ degrees when the zeroresponse partially coded FoV is considered.

The IBIS collimation system is made by using a passive shield. Its structural components are composed of a $1 \mathrm{~mm}$ thick tungsten hopper (placed on top of the detection systems) and a lead tube with a variable thickness extending up to the mask. A $1.5 \mathrm{~mm}$ thick lead door complements the shield in the direction opposite to the SPI (see Fig. A.1 bottom panel). The whole system, becoming increasingly transparent for energies above $200 \mathrm{keV}$, was designed to reduce the diffuse sky component of the instrument background.

The detection plane consists of two layers. The top, ISGRI, is optimized for the collection of photons up to energies of $\$ 500 \mathrm{keV}$ and is placed $90 \mathrm{~mm}$ above the other layer, PICsIT, which covers a higher energy range extending up to $10 \mathrm{MeV}$. Both detector arrays are surrounded by an active shield, the IBIS/Veto system, which is essential in order to reduce the cosmic-ray-induced instrument background.

\section{A.2.1. ISGRI}

IBIS/ISGRI (Lebrun et al. 2003) is the top detector layer of the IBIS telescope and is made of $163844 \times 4 \mathrm{~mm}$ and $2 \mathrm{~mm}$ thick CdTe pixels. It is sensitive to photons with energies between 15 and $800 \mathrm{keV}$ and retains a detection efficiency of nearly $100 \%$ for photons up to $\sim 100 \mathrm{keV}$. The effective area of ISGRI is reduced in the energy range $100 \mathrm{keV}-1 \mathrm{MeV}$, as it becomes increasingly optically thin to such radiation. In this energy range, photons begin to interact with the full volume of the detector pixels. The charge collection for interactions occurring at great depths in the detector is less efficient, complicating reconstruction of the incident photon energy. Nevertheless, ISGRI maintains good energy resolution up to $800 \mathrm{keV}$, thanks to the original software-based charge loss correction. The charge loss is reduced for photons arriving from highly off-axis sources, thus increasing the instrument performances for the study of such objects. The instrumental energy resolution toward the end of 2015 ranged from $15 \%$ at $60 \mathrm{keV}$ to $9 \%$ at $511 \mathrm{keV}$ (full width half maximum, FWHM). These values slowly worsen with time owing to the degradation of the detector gain, which is related to the evolution of charge collection efficiency driven by the long term irradiation of the detector in space (Caballero et al. 2013) and which becomes the principal cause for the current systematic uncertainties in the instrument calibrations. For the same reason, the characteristic low-energy threshold of the instrument increased from $15 \mathrm{keV}$ at launch to $22 \mathrm{keV}$ in 2015, at the same time becoming progressively less sharp. Simultaneous increase and smoothing of low threshold efficiency cut-off implies that photons with energies not far above $15 \mathrm{keV}$ can still be detected after more than $14 \mathrm{yr}$ in orbit, albeit with dramatically decreased efficiency.

The coded mask through which ISGRI usually observes the high-energy sky cannot be fully exploited when searching for impulsive events within the fully coded FoV when their location is not known. While the sensitivity for a source in a fixed location can be improved by using the coded mask pattern to reject about $50 \%$ of the background, this advantage is lost in a search for a new source, when there are additional trial factors. The conditions are different in the partially coded FoV, as a progressively smaller fraction of the detector is exposed through the coded mask holes and searches for short transients could be optimized by considering a smaller portion of the detector relevant for specific directions. This reduces the background, which is proportional to the total effective area used for the search. However, the instrument sensitivity is also reduced by exploring lower effective areas, rapidly approaching that of the SPI-ACS (see Figs. 4 and A.1 upper panel). We generally prefer to rely on the light curves built from the entire detector to search for impulsive events in the ISGRI data.

As the IBIS collimator tube is becoming increasingly transparent at energies above $\sim 200 \mathrm{keV}$, photons from directions that are up to 80 deg off-axis with respect to the satellite aim point can reach the ISGRI detectors, allowing this instrument to detect events occurring outside its FoV. Even soft events, with the bulk of photons released below $\sim 200 \mathrm{keV}$, can be detected in ISGRI despite the absorption by the IBIS shield and other satellite structures. For the very same reason, photons from soft events produce a highly contrasted pattern on the ISGRI detector plane that can be used to roughly constrain the source location (as is done with a higher precision when the event is recorded through the coded mask within the instrument FoV).

ISGRI is particularly well suited to searching for long transients, i.e., those associated with GRB afterglows, because the coded mask imaging allows us to better characterize the instrument background, as well as accurately subtracting the contribution of persistent sources from the data to probe the presence of faint transients.

\section{A.3. PICsIT}

IBIS/PICsIT (Labanti et al. 2003) is the bottom detector layer of the IBIS telescope and it is located $90 \mathrm{~mm}$ below ISGRI. It is made of $409630 \mathrm{~mm}$-thick CsI pixels, featuring a total collecting area of about $2900 \mathrm{~cm}^{2}$ and sensitive to photons with energies between $175 \mathrm{keV}$ and $10 \mathrm{MeV}$. In this energy range, the IBIS collimator tube is largely transparent and thus PICsIT can observe sources from all the directions that are not occulted by IBIS/Veto (i.e., with off-axis angles as large as $70 \mathrm{deg}$; see Sect. A.4). The 

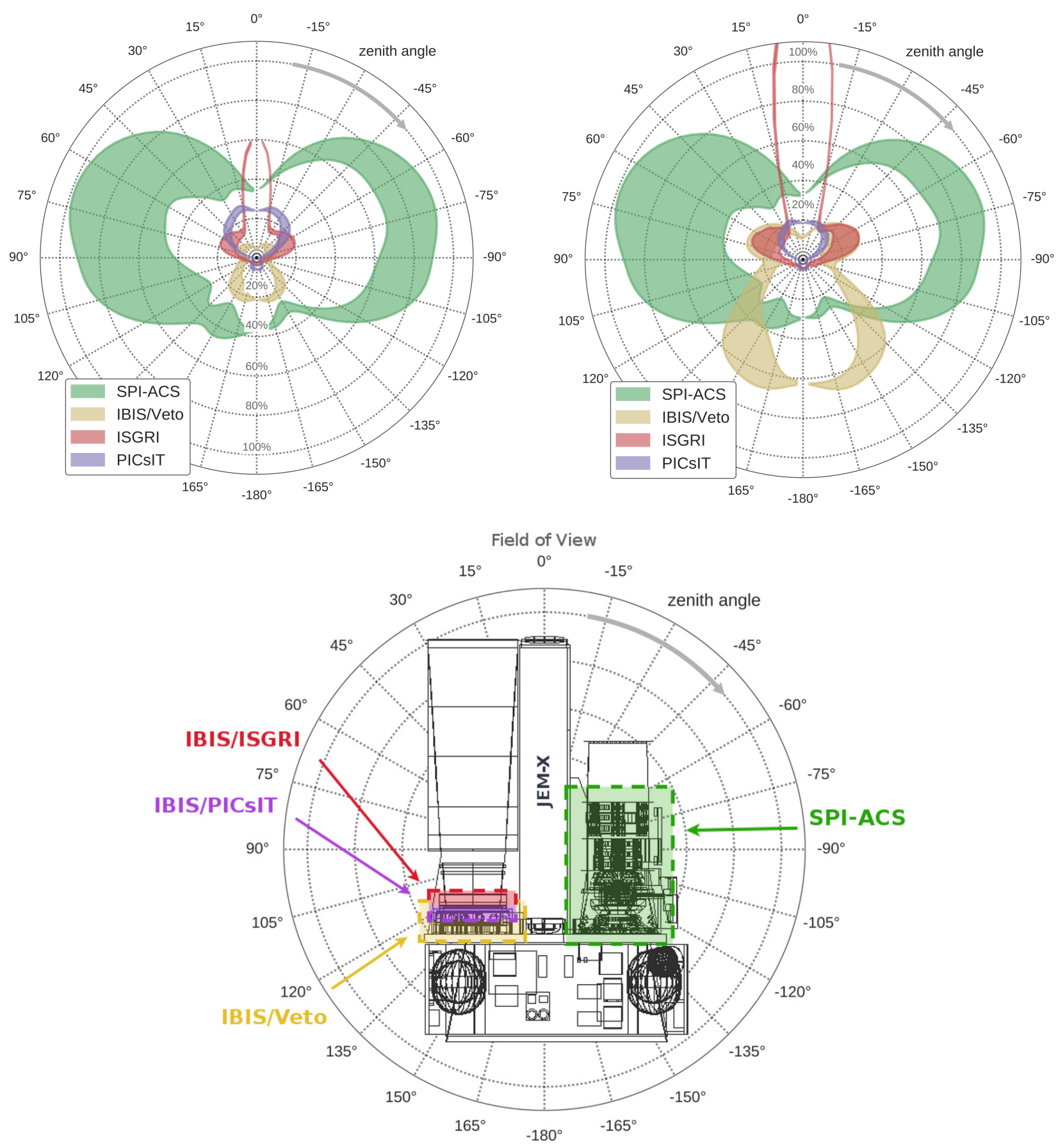

Fig. A.1. Top left panel: detection significances achievable on the entire sky by the omni-directional instruments on board INTEGRAL, together with those of the IBIS/ISGRI and IBIS/PICsIT (see Fig. 4 for a more detailed description of the imaging instrument sensitivity for observations within their FoVs). The detection significances have been normalized to the maximum value that can be obtained by the SPI-ACS in the most favorable orientation and considering a $1 \mathrm{~s}$ event whose emission is characterized by a Comptonized spectral model with $\alpha=-0.5$ and $E_{\mathrm{p}}=$ $600 \mathrm{keV}$. A shaded region is drawn for each instrument to represent how the detection significances change at different zenith angles (explicitly marked on the figure axes), also taking into account the dependence of the instrument sensitivity as a function of the azimuthal angles (not explicitly indicated in the figure). For each zenith angle, we computed the distribution of the significances for all relevant azimuthal angles; for clarity, we excluded from the shaping of the shaded regions the $10 \%$ worst and $10 \%$ best values. The satellite pointing direction is toward zenith angle 0 deg, while the axis pointing from SPI to IBIS is along the 90 deg direction. Top right panel: same as top left, but for an $8 \mathrm{~s}$ event whose emission is characterized by a Band spectral model (Band et al. 1993) with $\alpha=-1, E_{\mathrm{p}}=300 \mathrm{keV}$, and $\beta=-2.5$. Bottom panel: sketch of INTEGRAL derived from the INTEGRAL mass model (TIMM) showing the satellite pointing direction (zenith angle 0 deg), the axis connecting SPI to IBIS (zenith angle $90 \mathrm{deg}$ ), and the location of the different instruments.

effective area of the instrument slowly decreases as a function of off-axis angle, mainly due to the effect of the PICsIT planar geometry combined with the change in opacity of the shield and ISGRI detector plane. As the instrument coded mask opacity to hard X-ray photons is larger than that of the passive shield, PICsIT in principle collects more signal from sources outside the FoV rather than those closer to the satellite aim point (at odds with the case of ISGRI). This leads to a sensitivity increase for 
isolated bright impulsive events. In these cases, the background variability can often be neglected and its average level well constrained before and after the event without relying on the coded mask.

To evaluate the response of PICsIT to high-energy bursts from any sky direction, it is important to take into account the partial absorption of the corresponding radiation by the satellite structures. We thus performed Monte Carlo simulations using the INTEGRAL mass model previously described by Ferguson et al. (2003) and improving it through the inclusion of a more detailed IBIS mass model (Laurent et al. 2003). We validated our approach by comparing the results obtained for the detection of sources within the instrument FoV with the predictions of the PICsIT responses based on the most recent instrument calibrations provided by the instrument team.

\section{A.3.1. IBIS Compton mode}

When an incident photon undergoes Compton scattering in ISGRI, the scattered photon may be subsequently detected by PICsIT. It is also possible that a photon is Compton back-scattered in PICsIT and then detected in ISGRI. The electronics of IBIS identifies these nearly simultaneous interactions and records them in what is known as the Compton mode data (Ubertini et al. 2003). The effective area of the Compton mode is lower than that of both ISGRI and PICsIT, reaching $50 \mathrm{~cm}^{2}$ at $400 \mathrm{keV}$ for a $30 \mathrm{deg}$ off-axis source.

In principle, it is possible to use the Compton kinematics to constrain the photon arrival direction and reject spurious coincidences between independent ISGRI and PiCSIT events, which are the main background components. When searching for a known source, the Compton-mode sensitivity can be improved by excluding events incompatible with a given source location (Forot et al. 2007). The localization area for a single photon is primarily limited by the PICsIT energy resolution and can be as small as $5 \%$ of the sky for a $2 \mathrm{MeV}$ incident photon. However, its size strongly depends on the energies of incident and Compton scattered photons. For a typical background spectrum, only a small fraction of the interactions can be accurately localized: appropriate event selections provide an average localization area per photon of about $20 \%$ of the sky. Selections based on the event localization accuracy suppresses the background and improves the determination of source coordinates, but reduces the number of usable photons. The trade-off between the event localization accuracy and the number of usable events has to be evaluated on a case-by-case basis. Our results on LVT151012 apply to a wide range of conditions and should be considered representative in the case of weak transient sources.

In the case of brighter events, the Compton mode offers an important advantage, as it suffers much less telemetry stream saturation gaps than both ISGRI and PiCSIT. If the prompt emission associated with a GW event is bright enough to be detected in the IBIS Compton mode data, the localization of this event could be even more precise. We expect that an angular resolution better than $\sim 5 \mathrm{deg}$ can be achieved for a $1 \mathrm{~s}$ event characterized by a spectral energy distribution similar to that of a short-hard GRB and a fluence higher than $5 \times 10^{-5} \mathrm{erg} \mathrm{cm}^{-2}$ (assuming the event occurs in the area covered with the optimal sensitivity of the IBIS Compton mode). A GRB-like event yielding a $20 \sigma$ detection in the IBIS Compton mode would also give rise to a detection at a significance of at least $50 \sigma$ in SPI-ACS $(300 \sigma$ for most of the accessible source directions). We thus expect to exploit data collected by IBIS in Compton mode only when a very strong detection of the same event is reported in SPI-ACS, IBIS/ISGRI, and IBIS/PICsIT.

The potential of the IBIS Compton mode to localize and characterize bright transients from at least $50 \%$ of the sky was previously demonstrated by Marcinkowski et al. (2006, 2007), but has not been systematically exploited yet.

\section{A.4. IBIS/Veto}

The bottom and lateral sides of the IBIS detectors are surrounded by an active anti-coincidence shield, IBIS/Veto, which is made of $2 \mathrm{~cm}$ thick BGO crystals (Quadrini et al. 2003). The count rate of IBIS/Veto is continuously integrated every $8 \mathrm{~s}$ and transmitted to the ground, making this subsystem an efficient detector of GRBs (and of other $\gamma$-ray transient phenomena), albeit with a reduced sensitivity for events shorter than the nominal integration time.

We used Monte Carlo simulations exploiting the INTEGRAL mass model (Ferguson et al. 2003) to compute the response of IBIS/Veto (see Fig. A.1). We checked our results by using the observations of bright GRBs that were detected by Fermi/GBM. For a good match, we had to adjust the low-energy threshold of the IBIS/Veto system for which we have a limited description. The estimated discrepancy between the observed number of counts in the IBIS/Veto and those expected based on the GBM results is found in all cases to be $\$ 20 \%$.

IBIS/Veto is a particularly useful instrument to study sources with off-axis angles larger than $120 \mathrm{deg}$ where the sensitivity of ISGRI, PICsIT, and the IBIS Compton mode are low. At these angles, the coverage provided by the SPI-ACS is also limited (see Fig. 5). We also note that there is a relatively small fraction of the sky (about 15\%, depending on the source spectral energy distribution) for which the effective area of the IBIS/Veto is larger than that of the SPI-ACS. Owing to its lower background, the IBIS/Veto has a factor of 4 improved sensitivity compared to the SPI-ACS for the detection of impulsive events that are longer than $8 \mathrm{~s}$ and localized in some directions close to the opposite of satellite pointing axis (see Fig. A.1).

\section{A.5. SPI}

SPI is a $\gamma$-ray spectrometer made of 19 hexagonal high-purity germanium detectors. A tungsten coded aperture mask characterized by a hexagonal pattern is located $1.7 \mathrm{~m}$ above the detection plane of the instrument, thus allowing SPI to image a large region of the sky at once (the fully coded FoV radius is $\sim 8 \mathrm{deg}$ ) with a typical angular resolution of $2.5 \mathrm{deg}$.

The germanium detectors are surrounded by an active anticoincidence shield, SPI-ACS, forming a collimator tube and a bottom shield. SPI-ACS is made of 91 BGO (bismuth germanate, $\mathrm{Bi}_{4} \mathrm{Ge}_{3} \mathrm{O}_{12}$ ) scintillator crystals and is very efficient in preventing $\gamma$ rays from reaching the germanium detectors from every direction apart from the instrument's FoV (see Sect. A.5.1). SPI data can thus only be exploited to study sources within its FoV.

\section{A.5.1. SPI-ACS}

In addition to its main function of providing a veto signal for charged particles irradiating the SPI instrument, ACS is also able 
to provide the count rate corresponding to all impinging particles and high-energy photons. It can thus be used as a nearly omnidirectional detector of transient events with an effective area reaching $0.7 \mathrm{~m}^{2}$ at energies above $\sim 75 \mathrm{keV}$ and a time resolution of $50 \mathrm{~ms}$ (von Kienlin et al. 2003). The effective area of SPIACS is strongly affected by the opacity of materials, which are used for the INTEGRAL satellite structure and other instrument detectors. To estimate this opacity, we performed a number of
Monte Carlo particle transport simulations using both the SPI detector mass model (Sturner et al. 2003) and the INTEGRAL satellite mass model (TIMM; Ferguson et al. 2003). We verified that both models predicted similar count rates for the known impulsive events observed by SPI-ACS to within an accuracy of $20 \%$. For the detector response evaluation, we only made use of the TIMM as it known to include a more accurate description of spacecraft structures. 